\title{
THE HOPF ALGEBRA OF INTEGER BINARY RELATIONS
}

\author{
VINCENT PILAUD AND VIVIANE PONS
}

\begin{abstract}
We construct a Hopf algebra on integer binary relations that contains under the same roof several well-known Hopf algebras related to the permutahedra and the associahedra: the Malvenuto-Reutenauer algebra on permutations, the Loday-Ronco algebra on planar binary trees, and the Chapoton algebras on ordered partitions and on Schröder trees. We also derive from our construction new Hopf structures on intervals of the weak order on permutations and of the Tamari order on binary trees.
\end{abstract}

\section{Contents}

1. Integer binary relations 2

1.1. Weak order 2

1.2. Hopf algebra 2

2. Integer posets

3. Permutations, weak order intervals, and ordered partitions 10

3.1. Permutations and the Malvenuto-Reutenauer algebra 11

3.2. Weak order element, interval and face posets 11

3.3. Quotient algebras 13

$\begin{array}{lr}\text { 3.4. Subalgebras } & 17\end{array}$

4. Binary trees, Tamari intervals, and Schröder trees 21

4.1. Binary trees and the Loday-Ronco algebra 21

4.2. Tamari order element, interval and face posets $\quad 22$

4.3. Quotient algebras $\quad 25$

$\begin{array}{ll}\text { 4.4. Subalgebras } & 25\end{array}$

$\begin{array}{lr}\text { Acknowledgments } & 29\end{array}$

$\begin{array}{lr}\text { References } & 29\end{array}$

An integer binary relation is a binary relation on $[n]:=\{1, \ldots, n\}$ for some $n \in \mathbb{N}$. Integer posets are integer binary relations that are moreover posets (i.e. reflexive, antisymmetric and transitive). Many fundamental combinatorial objects (see Table 1 left) can be thought of as specific integer posets. This observation was used in [CPP17] to reinterpret classical lattice structures (see Table 1 middle) as specializations (subposets or sublattices) of a lattice structure called the weak order on posets. This interpretation enables to consider simultaneously all these specific integer posets and motivated the emergence of permutrees [PP18], which are combinatorial objects interpolating between permutations, binary trees, and Cambrian trees [CP17].

In this paper, we continue the exploration of the algebraic combinatorics of integer binary relations and integer posets, focussing on Hopf structures. We construct a Hopf algebra on integer binary relations where

(i) the product $\mathrm{R} \cdot \mathrm{S}$ of two relations $\mathrm{R}, \mathrm{S}$ is the sum of all relations that contain $\mathrm{R}$ at the beginning and $\mathrm{S}$ at the end as induced subrelations,

(ii) the coproduct $\triangle(\mathrm{R})$ of a relation $\mathrm{R}$ is the sum of the tensor products of the subrelations induced by $\mathrm{R}$ over all possible partitions $[n]=A \sqcup B$ that correspond to a total cut of $\mathrm{R}$.

We then reinterpret classical Hopf algebras [MR95, LR98, Cha00] (see Table 1 right) as specializations (quotients or subalgebras) of the integer poset algebra. Moreover, we obtain Hopf structures on the intervals of the weak order and on the intervals of the Tamari lattice, that remained undiscovered to the best of our knowledge.

VPi was partially supported by the French ANR grants SC3A (15 CE40 0004 01) and CAPPS (17 CE40 0018). 


\begin{tabular}{c|c|c} 
combinatorial object & lattice structure & Hopf algebra \\
\hline permutations & weak order & Malvenuto-Reutenauer algebra [MR95] \\
binary trees & Tamari lattice & Loday-Ronco algebra [LR98] \\
ordered partitions & facial weak order [KLN ${ }^{+} 01$, PR06, DHP18] & Chapoton algebra on ordered partitions [Cha00] \\
Schröder trees & facial Tamari order [PR06, DHP18] & Chapoton algebra on Schröder trees [Cha00] \\
weak order intervals & interval lattice of the weak order & NEW, see Sections 3.3.2 and 3.4.1 \\
Tamari order intervals & interval lattice of the Tamari lattice [CP15] & NEW, see Section 4.4
\end{tabular}

TABLE 1. Algebraic structures on classical combinatorial objects that can be reinterpreted as integer binary relations. See also Figure 3 for the connections between the Hopf algebras.

\section{INTEGER BINARY RELATIONS}

Our main object of focus are binary relations on integers. An integer (binary) relation of size $n$ is a binary relation on $[n]:=\{1, \ldots, n\}$, that is, a subset $\mathrm{R}$ of $[n]^{2}$. As usual, we write equivalently $(u, v) \in \mathrm{R}$ or $u \mathrm{R} v$, and similarly, we write equivalently $(u, v) \notin \mathrm{R}$ or $u R v$. Throughout the paper, all relations are implicitly assumed to be reflexive $(x \mathrm{R} x$ for all $x \in[n])$, although we often forget to include the diagonal $\{(u, u) \mid u \in[n]\}$ in our descriptions. We denote by $\mathcal{R}_{n}$ the set of all (reflexive) binary relations on $[n]$ and let $\mathcal{R}:=\bigsqcup_{n \geq 0} \mathcal{R}_{n}$.

1.1. Weak order. A lattice structure called the weak order on integer binary relations has been defined in [CPP17]. We recall its definition here as we will latter use this order to give a combinatorial description of the product.

Let $\mathrm{I}_{n}:=\left\{(a, b) \in[n]^{2} \mid a \leq b\right\}$ and $\mathrm{D}_{n}:=\left\{(b, a) \in[n]^{2} \mid a \leq b\right\}$. Observe that $\mathrm{I}_{n} \cup \mathrm{D}_{n}=[n]^{2}$ while $\mathrm{I}_{n} \cap \mathrm{D}_{n}=\{(a, a) \mid a \in[n]\}$. We say that the relation $\mathrm{R} \in \mathcal{R}_{n}$ is increasing (resp. decreasing) when $\mathrm{R} \subseteq \mathrm{I}_{n}$ (resp. $\mathrm{R} \subseteq \mathrm{D}_{n}$ ). The increasing and decreasing subrelations of an integer relation $\mathrm{R} \in$ $\mathcal{R}_{n}$ are the relations defined by:

$$
\mathrm{R}^{\text {Inc }}:=\mathrm{R} \cap \mathrm{I}_{n}=\{(a, b) \in \mathrm{R} \mid a \leq b\} \quad \text { and } \quad \mathrm{R}^{\mathrm{Dec}}:=\mathrm{R} \cap \mathrm{D}_{n}=\{(b, a) \in \mathrm{R} \mid a \leq b\} .
$$

In our pictures, we always represent an integer relation $\mathrm{R} \in \mathcal{R}_{n}$ as follows: we write the numbers $1, \ldots, n$ from left to right and we draw the increasing relations of $\mathrm{R}$ above in blue and the decreasing relations of $\mathrm{R}$ below in red. Although we only consider reflexive relations, we always omit the relations $(i, i)$ in the pictures (as well as in our explicit examples). See e.g. Figure 1.

Definition 1. The weak order on $\mathcal{R}_{n}$ is given by $\mathrm{R} \preccurlyeq \mathrm{S}$ if $\mathrm{R}^{\operatorname{lnc}} \supseteq \mathrm{S}^{\operatorname{lnc}}$ and $\mathrm{R}^{\text {Dec }} \subseteq \mathrm{S}^{\text {Dec }}$.

Note that the weak order is obtained by combining the refinement lattice on increasing subrelations with the coarsening lattice on decreasing subrelations. It explains the following statement.

Proposition 2. The weak order $\left(\mathcal{R}_{n}, \preccurlyeq\right)$ is a graded lattice whose meet and join are given by

$$
\mathrm{R} \wedge_{\mathcal{R}} \mathrm{S}=\left(\mathrm{R}^{\operatorname{lnc}} \cup \mathrm{S}^{\operatorname{lnc}}\right) \cup\left(\mathrm{R}^{\text {Dec }} \cap \mathrm{S}^{\text {Dec }}\right) \quad \text { and } \quad \mathrm{R} \vee_{\mathcal{R}} \mathrm{S}=\left(\mathrm{R}^{\operatorname{lnc}} \cap \mathrm{S}^{\operatorname{lnc}}\right) \cup\left(\mathrm{R}^{\text {Dec }} \cup \mathrm{S}^{\text {Dec }}\right) .
$$

1.2. Hopf algebra. We consider the vector space $\mathbf{k} \mathcal{R}:=\bigoplus_{n \geq 0} \mathbf{k} \mathcal{R}_{n}$ indexed by all integer binary relations of arbitrary size. We denote by $\left(\mathbb{F} \mathcal{R}_{R}\right)_{R \in \mathcal{R}}$ the standard basis of $\mathbf{k} \mathcal{R}$. In this section, we define a product and coproduct that endow $\mathbf{k} \mathcal{R}$ with a Hopf algebra structure.

We denote by $\mathrm{R}_{X}:=\left\{(i, j) \in[k]^{2} \mid x_{i} \mathrm{R} x_{k}\right\}$ the restriction of an integer relation $\mathrm{R} \in \mathcal{R}_{n}$ to a subset $X=\left\{x_{1}, \ldots, x_{k}\right\} \subseteq[n]$. Intuitively, it is just the restriction of the relation $\mathrm{R}$ to the subset $X$ which is then standardized to obtain a proper integer binary relation.

1.2.1. Product. The product that we define on binary integer relation generalizes the shifted shuffle of permutations: for $\mathrm{R} \in \mathcal{R}_{m}$ and $\mathrm{S} \in \mathcal{R}_{n}$, deleting the first $m$ values (resp. last $n$ values) in any relation of the shifted shuffle $\mathrm{R} \Psi \mathrm{S}$ yields the relation $\mathrm{S}$ (resp. R).

For $m, n \in \mathbb{N}$, we denote by $\overline{[n]}^{m}:=\{m+1, \ldots, m+n\}$ the interval $[n]$ shifted by $m$. For $m \in \mathbb{N}$ and $\mathrm{S} \in \mathcal{R}_{n}$, we denote by $\overline{\mathrm{S}}^{m}:=\{(m+i, m+j) \mid(i, j) \in \mathrm{S}\}$ the shifted relation. We also simply use $\overline{[n]}$ and $\overline{\mathrm{S}}$ when $m$ is clear from the context. 


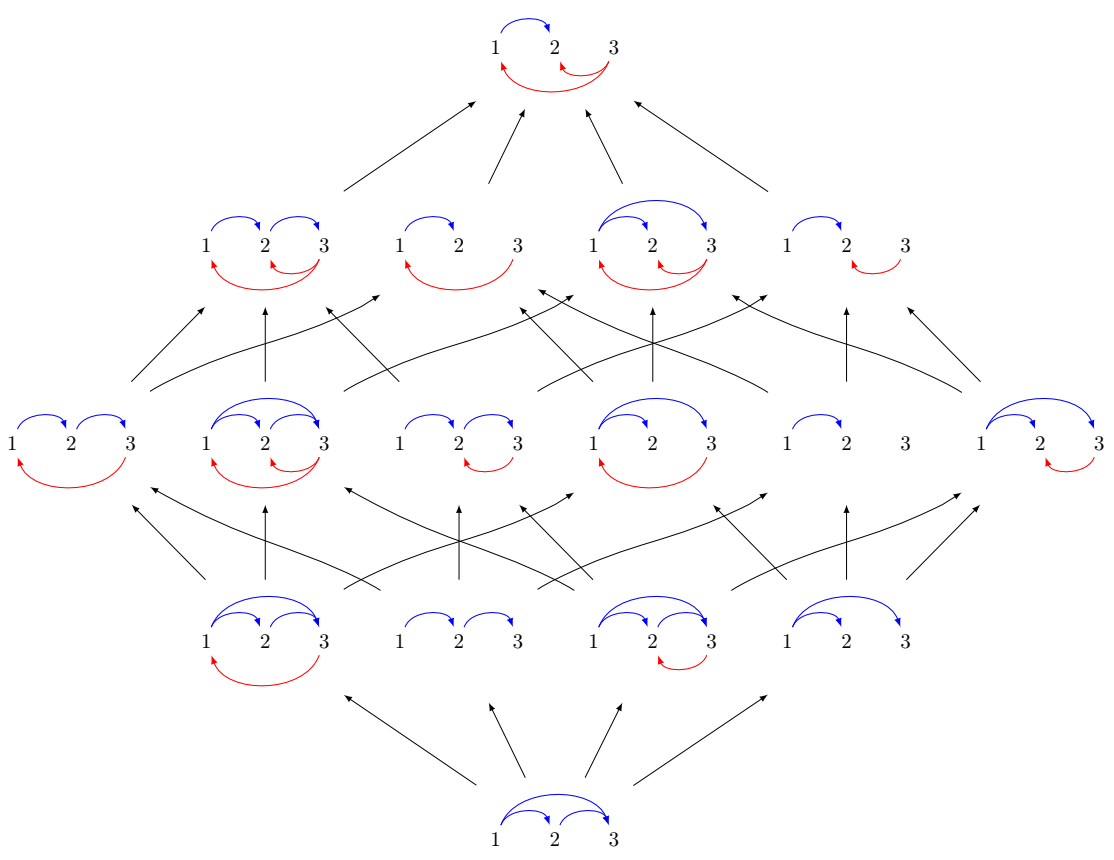

FiguRE 1. Interval corresponding to a product of relations.

For $\mathrm{R} \in \mathcal{R}_{m}$ and $\mathrm{S} \in \mathcal{R}_{n}$, we define $\mathrm{R} \backslash \mathrm{S}:=\mathrm{R} \cup \overline{\mathrm{S}} \cup([m] \times \overline{[n]})$ and $\mathrm{R} / \mathrm{S}:=\mathrm{R} \cup \overline{\mathrm{S}} \cup(\overline{[n]} \times[m])$.

Definition 3. For two relations $\mathrm{R} \in \mathcal{R}_{m}$ and $\mathrm{S} \in \mathcal{R}_{n}$, define the shifted shuffle $\mathrm{R} \overline{\mathrm{S}}$ as the set of relations $\mathrm{R} \sqcup \overline{\mathrm{S}} \sqcup \mathrm{I} \sqcup \mathrm{D}$ for all possible $\mathrm{I} \subseteq[\mathrm{m}] \times \overline{[n]}$ and $\mathrm{D} \subseteq \overline{[n]} \times[m]$.

Remark 4. Note that the shifted shuffle of $\mathrm{R} \in \mathcal{R}_{m}$ and $\mathrm{S} \in \mathcal{R}_{n}$ has cardinality $|\mathrm{R} \varpi \mathrm{S}|=2^{2 m n}$.

Example 5. For instance,

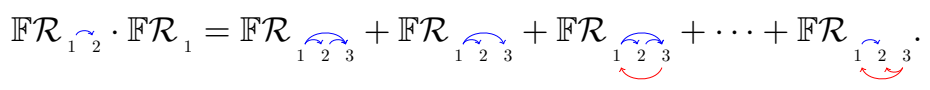

where the sum ranges over all relations in the interval of Figure 1.

Proposition 6. For $\mathrm{R} \in \mathcal{R}_{m}, \mathrm{~S} \in \mathcal{R}_{n}$ and $\mathrm{T} \in \mathcal{R}_{m+n}$, we have

$$
\mathrm{T} \in \mathrm{R} \overline{\mathrm{S}} \quad \Longleftrightarrow \quad \mathrm{T}_{[m]}=\mathrm{R} \text { and } \mathrm{T}_{\overline{[n]}}=\mathrm{S} \quad \Longleftrightarrow \quad \mathrm{R} \backslash \mathrm{S} \preccurlyeq \mathrm{T} \preccurlyeq \mathrm{R} / \mathrm{S} .
$$

Proof. The first equivalence is immediate. Assume now that $\mathrm{T}=\mathrm{R} \sqcup \overline{\mathrm{S}} \sqcup \mathrm{I} \sqcup \mathrm{D}$ for some $\mathrm{I} \subseteq[m] \times \overline{[n]}$ and $\mathrm{D} \subseteq \overline{[n]} \times[m]$. Then $\mathrm{T}^{\mathrm{Inc}}=\mathrm{R}^{\operatorname{lnc}} \cup \overline{\mathrm{S}}^{\operatorname{Inc}} \cup \mathrm{I} \subseteq \mathrm{R}^{\operatorname{lnc}} \cup \overline{\mathrm{S}}^{\operatorname{lnc}} \cup([m] \times \overline{[n]})=(\mathrm{R} \backslash \mathrm{S})^{\operatorname{lnc}}$ and $\mathrm{T}^{\mathrm{Dec}}=\mathrm{R}^{\mathrm{Dec}} \cup \overline{\mathrm{S}}^{\mathrm{Dec}} \cup \mathrm{D} \supseteq \mathrm{R}^{\mathrm{Dec}} \cup \overline{\mathrm{S}}^{\mathrm{Dec}}=(\mathrm{R} \backslash \mathrm{S})^{\text {Dec }}$ so that $\mathrm{R} \backslash \mathrm{S} \preccurlyeq \mathrm{T}$. Similarly $\mathrm{T} \preccurlyeq \mathrm{R} / \mathrm{S}$. Conversely, if $\mathrm{R} \backslash \mathrm{S} \preccurlyeq \mathrm{T} \preccurlyeq \mathrm{R} / \mathrm{S}$, then $\mathrm{R}=\mathrm{R} \backslash \mathrm{S}_{[m]} \preccurlyeq \mathrm{T}_{[m]} \preccurlyeq \mathrm{R} / \mathrm{S}_{[m]}=\mathrm{R}$, thus $\mathrm{T}_{[m]}=\mathrm{R}$. Similarly $\mathrm{T}_{[n]}=\mathrm{S}$.

Definition 7. The product of two integer relations $\mathrm{R} \in \mathcal{R}_{m}$ and $\mathrm{S} \in \mathcal{R}_{n}$ is

$$
\mathbb{F} \mathcal{R}_{\mathrm{R}} \cdot \mathbb{F} \mathcal{R}_{\mathrm{S}}:=\sum_{\mathrm{T} \in \mathrm{R} \varpi \mathrm{S}} \mathbb{F} \mathcal{R}_{\mathrm{T}}
$$

Proposition 8. The product $\cdot$ defines an associative graded algebra structure on $\mathbf{k} \mathcal{R}$.

Proof. For $\mathrm{R} \in \mathcal{R}_{m}$ and $\mathrm{S} \in \mathcal{R}_{n}$, all relations in $\mathrm{T} \overline{\mathrm{S}} \mathrm{S}$ belong to $\mathcal{R}_{m+n}$ by definition. Moreover, for $\mathrm{R} \in \mathcal{R}_{m}, \mathrm{~S} \in \mathcal{R}_{n}, \mathrm{~T} \in \mathcal{R}_{o}$, Proposition 6 ensures that the relations in (R山 $\left.\mathrm{S}\right) \varpi \mathrm{T}$ and in $\mathrm{R} \varpi(\mathrm{S} \varpi \mathrm{T})$ are the relations $\mathrm{U} \in \mathcal{R}_{m+n+o}$ such that $\mathrm{U}_{[m]}=\mathrm{T}, \mathrm{U}_{\overline{[n]}^{m}}=\mathrm{S}$ and $\mathrm{U}_{\overline{[o]}^{m+n}}=\mathrm{T}$. 
By Proposition 6, we know that this product can be interpreted as a sum over an interval. We now prove a property slightly more general.

Proposition 9. The product of two intervals is an interval: for $\mathrm{R} \preccurlyeq \mathrm{R}^{\prime}$ in $\mathcal{R}_{m}$ and $\mathrm{S} \preccurlyeq \mathrm{S}^{\prime}$ in $\mathcal{R}_{n}$,

$$
\left(\sum_{\mathrm{R} \preccurlyeq \mathrm{U} \preccurlyeq \mathrm{R}^{\prime}} \mathbb{F} \mathcal{R}_{\mathrm{U}}\right) \cdot\left(\sum_{\mathrm{S} \preccurlyeq \mathrm{V} \preccurlyeq \mathrm{S}^{\prime}} \mathbb{F} \mathcal{R}_{\mathrm{V}}\right)=\left(\sum_{\mathrm{R} \backslash \mathrm{S} \preccurlyeq \mathrm{T} \preccurlyeq \mathrm{R}^{\prime} / \mathrm{S}^{\prime}} \mathbb{F} \mathcal{R}_{\mathrm{T}}\right) .
$$

Proof. We have

$$
\left(\sum_{\mathrm{R} \preccurlyeq \mathrm{U} \preccurlyeq \mathrm{R}^{\prime}} \mathbb{F} \mathcal{R}_{\mathrm{U}}\right) \cdot\left(\sum_{\mathrm{S} \preccurlyeq \mathrm{V} \preccurlyeq \mathrm{S}^{\prime}} \mathbb{F} \mathcal{R}_{\mathrm{V}}\right)=\sum_{\substack{\mathrm{R} \preccurlyeq \mathrm{U} \preccurlyeq \mathrm{R}^{\prime} \\ \mathrm{S} \preccurlyeq \mathrm{V} \preccurlyeq \mathrm{S}^{\prime}}} \sum_{\mathrm{T} \in \mathrm{U} \varpi \mathrm{V}} \mathbb{F} \mathcal{R}_{\mathrm{T}} \cdot
$$

First note that all coefficients are equal to 1 . Indeed, any relation $\mathrm{T}$ of the sum belongs to exactly one set $\mathrm{U} \overline{\mathrm{V}}$ as $\mathrm{U}$ and $\mathrm{V}$ are uniquely defined by $\mathrm{U}=\mathrm{T}_{[m]}$ and $\mathrm{V}=\mathrm{T}_{\overline{[n]}}$. The only thing to prove is then

$$
\left\{T \in \mathcal{R} \mid \mathrm{R} \backslash \mathrm{S} \preccurlyeq T \preccurlyeq \mathrm{R}^{\prime} / \mathrm{S}^{\prime}\right\}=\bigsqcup_{\substack{\mathrm{R} \preccurlyeq \mathrm{U} \preccurlyeq \mathrm{R}^{\prime} \\ \mathrm{S} \preccurlyeq \mathrm{V} \preccurlyeq \mathrm{S}^{\prime}}}\{T \in \mathcal{R} \mid \mathrm{U} \backslash \mathrm{V} \preccurlyeq T \preccurlyeq \mathrm{U} / \mathrm{V}\}
$$

where the union on the right is disjoint. Let us call $A$ the set on the left and $B$ the set on the right. It is clear that $B \subseteq A$. Indeed, for $T \in B$, we have $\mathrm{U} \backslash \mathrm{V} \preccurlyeq T \preccurlyeq \mathrm{U} / \mathrm{V}$ for some $\mathrm{R} \preccurlyeq \mathrm{U} \preccurlyeq \mathrm{R}^{\prime}$ and $\mathrm{S} \preccurlyeq \mathrm{V} \preccurlyeq \mathrm{S}^{\prime}$ : this gives directly $\mathrm{R} \backslash \mathrm{S} \preccurlyeq \mathrm{T} \preccurlyeq \mathrm{R}^{\prime} / \mathrm{S}^{\prime}$. Conversely, let $\mathrm{T} \in \mathcal{R}$ be such that $\mathrm{R} \backslash \mathrm{S} \preccurlyeq \mathrm{T} \preccurlyeq \mathrm{R}^{\prime} / \mathrm{S}^{\prime}$. This means $\left(\mathrm{R}^{\prime} / \mathrm{S}^{\prime}\right)^{\operatorname{lnc}} \subseteq \mathrm{T}^{\operatorname{lnc}} \subseteq(\mathrm{R} \backslash \mathrm{S})^{\operatorname{lnc}}$ and $\left(\mathrm{R}^{\prime} / \mathrm{S}^{\prime}\right)^{\text {Dec }} \supseteq \mathrm{T}^{\text {Dec }} \supseteq(\mathrm{R} \backslash \mathrm{S})^{\text {Dec }}$. This is still true if the relations are restricted to $[m]$ (resp. $\overline{[n]}$ ). For $\mathrm{U}:=\mathrm{T}_{[m]}$ and $\mathrm{V}:=\mathrm{T}_{\overline{[n]}}$, we get that $(\mathrm{R} \backslash \mathrm{S})_{[m]}=\mathrm{R} \preccurlyeq \mathrm{U} \preccurlyeq \mathrm{R}^{\prime}=\left(\mathrm{R}^{\prime} / \mathrm{S}^{\prime}\right)_{[m]}$ and $(\mathrm{R} \backslash \mathrm{S})_{\overline{[n]}}=\mathrm{S} \preccurlyeq \mathrm{V} \preccurlyeq \mathrm{S}^{\prime}=\left(\mathrm{R}^{\prime} / \mathrm{S}^{\prime}\right)_{\overline{[n]}}$. Now $\mathrm{T}=\mathrm{U} \cup \mathrm{V} \cup I$ with $I \subseteq([m] \times \overline{[n]}) \cup(\overline{[n]} \times[m])$ which means $T \in \mathrm{U} \overline{\mathrm{V}}$.

1.2.2. Coproduct. We now define a coproduct on integer relations using total cuts.

Definition 10. A total cut $(X, Y)$ of a relation $\mathrm{T} \in \mathcal{R}_{p}$ is a partition $[p]=X \sqcup Y$ such that $x \mathrm{~T} y$ and $y$ T $x$ for all $x \in X$ and $y \in Y$. For two relations $\mathrm{R} \in \mathcal{R}_{m}$ and $\mathrm{S} \in \mathcal{R}_{n}$, define the convolution $\mathrm{R} \star \mathrm{S}$ as the set of relations $\mathrm{T} \in \mathcal{R}_{m+n}$ which admit a total cut $(X, Y)$ such that $\mathrm{T}_{X}=\mathrm{R}$ and $\mathrm{T}_{Y}=\mathrm{S}$.

Remark 11. Note that the convolution of $\mathrm{R} \in \mathcal{R}_{m}$ and $\mathrm{S} \in \mathcal{R}_{n}$ has cardinality $|\mathrm{R} \star \mathrm{S}|=\left(\begin{array}{c}m+n \\ m\end{array}\right)$.

Definition 12. The coproduct of an integer relation $\mathrm{T} \in \mathcal{R}$ is

$$
\triangle\left(\mathbb{F} \mathcal{R}_{\mathrm{T}}\right):=\sum_{\mathrm{T} \in \mathrm{R} \star \mathrm{S}} \mathbb{F} \mathcal{R}_{\mathrm{R}} \otimes \mathbb{F} \mathcal{R}_{\mathrm{S}}
$$

Example 13. For instance,

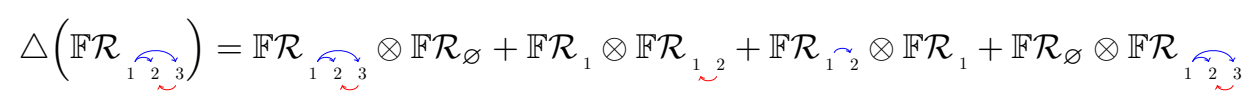

where the terms in the coproduct arise from the total cuts $(\{1,2,3\}, \varnothing),(\{1\},\{2,3\}),(\{1,3\},\{2\})$, and $(\varnothing,\{1,2,3\})$.

Proposition 14. The coproduct $\triangle$ defines a coassociative graded coalgebra structure on $\mathbf{k} \mathcal{R}$.

Proof. For $\mathrm{R} \in \mathcal{R}_{m}$ and $\mathrm{S} \in \mathcal{R}_{n}$, all relations in $\mathrm{T} \star \mathrm{S}$ belong to $\mathcal{R}_{m+n}$ by definition. Moreover, for $\mathrm{R} \in \mathcal{R}_{m}, \mathrm{~S} \in \mathcal{R}_{n}, \mathrm{~T} \in \mathcal{R}_{o}$, the relations in $(\mathrm{R} \star \mathrm{S}) \star \mathrm{T}$ and in $\mathrm{R} \star(\mathrm{S} \star \mathrm{T})$ are precisely the relations $\mathrm{U} \in \mathcal{R}_{m+n+o}$ such that there is a partition $[m+n+o]=X \sqcup Y \sqcup Z$ such that

- $\{(x, y),(x, z),(y, z)\} \subseteq \mathrm{U}$ and $\{(y, x),(z, x),(z, y)\} \cap \mathrm{U}=\varnothing$ for all $x \in X, y \in Y, z \in Z$,

- $\mathrm{U}_{X}=\mathrm{T}, \mathrm{U}_{Y}=\mathrm{S}$ and $\mathrm{U}_{Z}=\mathrm{T}$. 
1.2.3. Hopf algebra. We now combine the algebra and coalgebra structures on $\mathbf{k} \mathcal{R}$ to a Hopf algebra. Recall that a combinatorial Hopf algebra is a combinatorial vector space endowed with an associative product $\cdot$ and a coassociative coproduct $\triangle$ which satisfy the compatibility relation $\triangle\left(\mathbb{F} \mathcal{R}_{\mathrm{R}} \cdot \mathbb{F} \mathcal{R}_{\mathrm{S}}\right)=\triangle\left(\mathbb{F} \mathcal{R}_{\mathrm{R}}\right) \cdot \triangle\left(\mathbb{F} \mathcal{R}_{\mathrm{S}}\right)$, where the last product is defined componentwise by $(a \otimes b) \cdot(c \otimes d)=(a \cdot c) \otimes(b \cdot d)$.

Proposition 15. The product $\cdot$ of Definition 7 and the coproduct $\triangle$ of Definition 12 endow $\mathbf{k} \mathcal{R}$ with a Hopf algebra structure.

Example 16. Before giving the formal proof, let us illustrate on an example the compatibility relation $\triangle\left(\mathbb{F} \mathcal{R}_{\mathrm{R}} \cdot \mathbb{F} \mathcal{R}_{\mathrm{S}}\right)=\triangle\left(\mathbb{F} \mathcal{R}_{\mathrm{R}}\right) \cdot \triangle\left(\mathbb{F} \mathcal{R}_{\mathrm{S}}\right)$. For that, let $\mathrm{R}$ and $\mathrm{S}$ both be the unique relation of size 1. On the one hand, we have:

$$
\begin{aligned}
\triangle\left(\mathbb{F} \mathcal{R}_{1}\right) \cdot \triangle\left(\mathbb{F} \mathcal{R}_{1}\right) & =\left(\mathbb{F} \mathcal{R}_{1} \otimes \mathbb{F} \mathcal{R}_{\varnothing}+\mathbb{F} \mathcal{R}_{\varnothing} \otimes \mathbb{F} \mathcal{R}_{1}\right) \cdot\left(\mathbb{F} \mathcal{R}_{1} \otimes \mathbb{F} \mathcal{R}_{\varnothing}+\mathbb{F} \mathcal{R}_{\varnothing} \otimes \mathbb{F} \mathcal{R}_{1}\right) \\
& =\left(\mathbb{F} \mathcal{R}_{1} \cdot \mathbb{F} \mathcal{R}_{1}\right) \otimes \mathbb{F} \mathcal{R}_{\varnothing}+2\left(\mathbb{F} \mathcal{R}_{1} \otimes \mathbb{F} \mathcal{R}_{1}\right)+\mathbb{F} \mathcal{R}_{\varnothing} \otimes\left(\mathbb{F} \mathcal{R}_{1} \cdot \mathbb{F} \mathcal{R}_{1}\right)
\end{aligned}
$$

with

$$
\mathbb{F} \mathcal{R}_{1} \cdot \mathbb{F} \mathcal{R}_{1}=\mathbb{F} \mathcal{R}_{12}+\mathbb{F} \mathcal{R}_{r_{2}}+\mathbb{F} \mathcal{R}_{\sim_{\sim 2}^{2}}+\mathbb{F} \mathcal{R}_{\sim_{\sim}^{\sim}}^{\sim}
$$

On the other hand, we have:

$$
\begin{aligned}
& \triangle\left(\mathbb{F} \mathcal{R}_{1} \cdot \mathbb{F} \mathcal{R}_{1}\right)=\triangle\left(\mathbb{F} \mathcal{R}_{12}+\mathbb{F} \mathcal{R}_{r_{2}}+\mathbb{F} \mathcal{R}_{12}+\mathbb{F} \mathcal{R}_{\sim_{2} \sim_{2}}\right) \\
& =\triangle\left(\mathbb{F} \mathcal{R}_{1} 2_{2}\right)+\triangle\left(\mathbb{F} \mathcal{R}_{\sim_{2}}\right)+\triangle\left(\mathbb{F} \mathcal{R}_{1_{2}}\right)+\triangle\left(\mathbb{F} \mathcal{R}_{\sim_{\sim_{2}}}\right) \\
& =\left(\mathbb{F} \mathcal{R}_{12} \otimes \mathbb{F} \mathcal{R}_{\varnothing}+\mathbb{F} \mathcal{R}_{\varnothing} \otimes \mathbb{F} \mathcal{R}_{11_{2}}\right)+\left(\mathbb{F} \mathcal{R}_{r_{2}} \otimes \mathbb{F} \mathcal{R}_{\varnothing}+\mathbb{F} \mathcal{R}_{1} \otimes \mathbb{F} \mathcal{R}_{1}+\mathbb{F} \mathcal{R}_{\varnothing} \otimes \mathbb{F} \mathcal{R}_{r_{2}}\right) \\
& +\left(\mathbb{F} \mathcal{R}_{\sim_{\sim}^{2}} \otimes \mathbb{F} \mathcal{R}_{\varnothing}+\mathbb{F} \mathcal{R}_{1} \otimes \mathbb{F} \mathcal{R}_{1}+\mathbb{F} \mathcal{R}_{\varnothing} \otimes \mathbb{F} \mathcal{R}_{\sim_{\sim^{2}}}\right)+\left(\mathbb{F} \mathcal{R}_{\sim_{\sim_{\sim}^{2}}}^{\sim} \otimes \mathbb{F} \mathcal{R}_{\varnothing}+\mathbb{F} \mathcal{R}_{\varnothing} \otimes \mathbb{F} \mathcal{R}_{\sim_{\sim} \sim_{2}}\right) \\
& =\left(\mathbb{F} \mathcal{R}_{1_{2}}+\mathbb{F} \mathcal{R}_{\Gamma_{2}}+\mathbb{F} \mathcal{R}_{\sim_{2}^{2}}+\mathbb{F} \mathcal{R}_{\sim_{\sim_{2}}}\right) \otimes \mathbb{F} \mathcal{R}_{\varnothing}+2\left(\mathbb{F} \mathcal{R}_{1} \otimes \mathbb{F} \mathcal{R}_{1}\right) \\
& +\mathbb{F} \mathcal{R}_{\varnothing} \otimes\left(\mathbb{F} \mathcal{R}_{12}+\mathbb{F} \mathcal{R}_{\sim_{2}}+\mathbb{F} \mathcal{R}_{\sim_{\sim 2}}+\mathbb{F} \mathcal{R}_{\sim_{\sim 2}}^{\sim}\right) \text {. }
\end{aligned}
$$

Therefore

$$
\triangle\left(\mathbb{F} \mathcal{R}_{1} \cdot \mathbb{F} \mathcal{R}_{1}\right)=\triangle\left(\mathbb{F} \mathcal{R}_{1}\right) \cdot \triangle\left(\mathbb{F} \mathcal{R}_{1}\right) .
$$

Proof of Proposition 15. We have

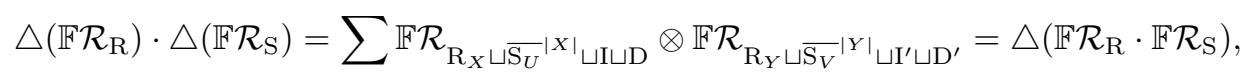

where the sum ranges over all total cuts $(X, Y)$ of $\mathrm{R}$ and $(U, V)$ of $\mathrm{S}$ and all relations

$$
\mathrm{I} \subseteq[|X|] \times \overline{[|U|]}^{|X|}, \quad \mathrm{D} \subseteq \overline{[|U|]}^{|X|} \times[|X|], \quad \mathrm{I}^{\prime} \subseteq[|Y|] \times \overline{[|V|]}^{|Y|} \quad \text { and } \quad \mathrm{D}^{\prime} \subseteq \overline{[|V|]}^{|Y|} \times[|Y|] .
$$

The first equality directly follows from the definitions. For the second equality, observe that for any $\mathrm{T} \in \mathrm{R} \overline{\mathrm{S}}$, the total cuts of $\mathrm{T}$ are precisely of the form $\left(X \sqcup \bar{U}^{m}, Y \sqcup \bar{V}^{m}\right)$ where $(X, Y)$ is a total cut of $\mathrm{R}$ and $(U, V)$ is a total cut of $\mathrm{S}$ such that $X \times \bar{V}^{m}$ and $Y \times \bar{U}^{m}$ are both subsets of $\mathrm{T}$ while $\bar{V}^{m} \times X$ and $\bar{U}^{m} \times Y$ are both subsets of the complement of T.

1.2.4. Multiplicative bases. In this section, we describe multiplicative bases of $\mathbf{k} \mathcal{R}$ and study the indecomposable elements of $\mathcal{R}$ for these bases. For a relation $\mathrm{R} \in \mathcal{R}$, we define

$$
\mathbb{E} \mathcal{R}^{\mathrm{R}}=\sum_{\mathrm{R} \preccurlyeq \mathrm{R}^{\prime}} \mathbb{F} \mathcal{R}_{\mathrm{R}^{\prime}} \quad \text { and } \quad \mathbb{H} \mathcal{R}^{\mathrm{R}}=\sum_{\mathrm{R}^{\prime} \preccurlyeq \mathrm{R}} \mathbb{F} \mathcal{R}_{\mathrm{R}^{\prime}}
$$

Example 17. For instance, $\quad \mathbb{E} \mathcal{R}^{1{ }^{2}}=\mathbb{F} \mathcal{R}_{12}+\mathbb{F} \mathcal{R}_{\sim_{\sim}^{2}} \quad$ and $\quad \mathbb{H} \mathcal{R}^{1{ }^{2}}=\mathbb{F} \mathcal{R}_{12}+\mathbb{F} \mathcal{R}_{\widetilde{1}_{2}}$.

Proposition 18. The sets $\left(\mathbb{E}^{\mathrm{R}}\right)_{\mathrm{R} \in \mathcal{R}}$ and $\left(\mathbb{H} \mathcal{R}^{\mathrm{R}}\right)_{\mathrm{R} \in \mathcal{R}}$ form multiplicative bases of $\mathbf{k} \mathcal{R}$ with

$$
\mathbb{E} \mathcal{R}^{\mathrm{R}} \cdot \mathbb{E} \mathcal{R}^{\mathrm{S}}=\mathbb{E} \mathcal{R}^{\mathrm{R} \backslash \mathrm{S}} \quad \text { and } \quad \mathbb{H} \mathcal{R}^{\mathrm{R}} \cdot \mathbb{H} \mathcal{R}^{\mathrm{S}}=\mathbb{H} \mathcal{R}^{\mathrm{R} / \mathrm{S}} .
$$

Proof. First note that the elements of $\left(\mathbb{E}^{\mathrm{R}}\right)_{\mathrm{R} \in \mathcal{R}}$ (resp. $\left(\mathbb{H} \mathcal{R}^{\mathrm{R}}\right)_{\mathrm{R} \in \mathcal{R}}$ ) are linearly independent: each element $\mathbb{E}^{\mathrm{R}}$ contains a leading term $\mathbb{F} \mathcal{R}_{\mathrm{R}}$ and so the transition matrix is triangular. The product formula is a direct consequence of Proposition 9. 
Example 19. For instance,

$$
\begin{aligned}
& \mathbb{E} \mathcal{R}^{1{ }^{2}} \cdot \mathbb{E} \mathcal{R}^{1}=\left(\mathbb{F} \mathcal{R}_{12}+\mathbb{F}_{\mathcal{R}_{1}{ }_{2}}\right) \cdot \mathbb{F} \mathcal{R}_{1} \\
& =\mathbb{F} \mathcal{R}_{12} \cdot \mathbb{F} \mathcal{R}_{1}+\mathbb{F} \mathcal{R}_{1_{2}^{2}} \cdot \mathbb{F} \mathcal{R}_{1}
\end{aligned}
$$

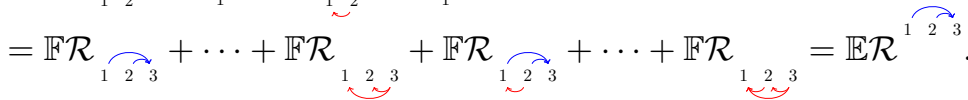

Note that even though $\mathbb{E} \mathcal{R}$ and $\mathbb{H} \mathcal{R}$ have very simple definitions for the product, the definition of the coproduct is now more complicated than on $\mathbb{F} \mathcal{R}$. In particular, we now have some coefficients greater than 1 which appear as in the example below.

Example 20. For instance,

$$
\begin{aligned}
& \Delta\left(\mathbb{E} \mathcal{R}^{{ }^{{ }^{2}}}\right)=\Delta\left(\mathbb{F} \mathcal{R}_{1_{2}}^{\sim_{2}}+\mathbb{F} \mathcal{R}_{1_{2}}^{\sim_{2}}+\mathbb{F} \mathcal{R}_{12}+\mathbb{F} \mathcal{R}_{12}\right)
\end{aligned}
$$

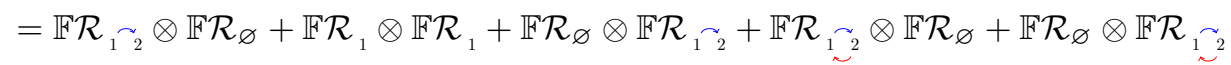

$$
\begin{aligned}
& +\mathbb{F} \mathcal{R}_{1_{2}} \otimes \mathbb{F} \mathcal{R}_{\varnothing}+\mathbb{F} \mathcal{R}_{\varnothing} \otimes \mathbb{F} \mathcal{R}_{1_{2}}+\mathbb{F} \mathcal{R}_{1_{2}} \otimes \mathbb{F} \mathcal{R}_{\varnothing}+\mathbb{F} \mathcal{R}_{1} \otimes \mathbb{F} \mathcal{R}_{1}+\mathbb{F} \mathcal{R}_{\varnothing} \otimes \mathbb{F} \mathcal{R}_{12} \\
& =\mathbb{E} \mathcal{R}^{{ }^{12}} \otimes \mathbb{E} \mathcal{R}^{\varnothing}+2\left(\mathbb{E} \mathcal{R}^{1} \otimes \mathbb{E} \mathcal{R}^{1}\right)+\widetilde{E} \mathcal{R}^{\varnothing} \otimes \mathbb{E} \mathcal{R}^{{ }^{1{ }^{2}}} \text {. }
\end{aligned}
$$

Definition 21. We say that a relation $\mathrm{T}$ is under-indecomposable (resp. over-indecomposable) if there is no $\mathrm{R}$ and $\mathrm{S}$ in $\mathcal{R}$ with $|\mathrm{R}| \geq 1$ and $|\mathrm{S}| \geq 1$ such that $\mathrm{T}=\mathrm{R} \backslash \mathrm{S}$ (resp. $\mathrm{T}=\mathrm{R} / \mathrm{S}$ ).

Proposition 22. The algebra $\mathbf{k} \mathcal{R}$ is freely generated by the elements $\mathbb{E} \mathcal{R}^{\mathrm{T}}$ where $\mathrm{T}$ is underindecomposable (resp. by the elements $\mathbb{H} \mathcal{R}^{\mathrm{T}}$ where $\mathrm{T}$ is over-indecomposable).

We will prove this proposition only for $\mathbb{E} \mathcal{R}^{\mathrm{T}}$. Besides, we will work solely with the notion of under-indecomposable which we will simply call indecomposable in the rest of paper when there is no ambiguity. The proof of Proposition 22 relies on the results of [LR06] on the (co-)freeness of (co-)associative algebras.

Definition 23 ([LR06, p. 7]). A unital infinitesimal bialgebra $(\mathcal{H}, \cdot, \mathbf{\Delta})$ is a vector space $\mathcal{H}$ equipped with a unital associative product · and a counital coassociative coproduct $\boldsymbol{\Delta}$ which are related by the unital infinitesimal relation:

$$
\overline{\mathbf{\Delta}}(x \cdot y)=(x \otimes 1) \cdot \overline{\mathbf{\Delta}}(y)+\overline{\mathbf{\Delta}}(x) \cdot(1 \otimes y)+x \otimes y,
$$

where the product $\cdot$ on $\mathcal{H} \otimes \mathcal{H}$ and the reduced coproduct $\mathbf{\boldsymbol { \Lambda }}$ are given by

$$
(x \otimes y) \cdot\left(x^{\prime} \otimes y^{\prime}\right)=\left(x \cdot x^{\prime}\right) \otimes\left(y \cdot y^{\prime}\right), \quad \text { and } \quad \overline{\mathbf{\Lambda}}(x):=\mathbf{\Delta}(x)-(x \otimes 1+1 \otimes x) .
$$

Note that this is not the classical compatibility relation satisfied by the product $\cdot$ and the coproduct $\triangle$ of a Hopf algebra. In particular, $(\mathcal{R}, \cdot, \triangle)$ is not a unital inifinesimal bialgebra. Nevertheless we will prove that for another coproduct $\boldsymbol{\Lambda}$, then $(\mathcal{R}, \cdot, \boldsymbol{\Lambda})$ is a unital inifinesimal bialgebra. We can then use the main result of [LR06].

Theorem 24 ([LR06, p. 2]). Any graded unital infinitesimal bialgebra is isomorphic to the noncommutative polynomials algebra equipped with the deconcatenation coproduct.

The isomorphism is explicit. Each element $x$ of $\mathcal{H}$ can be written uniquely as a product $x=x_{1} \cdot x_{2} \ldots \cdot x_{k}$ such that the elements $x_{i}$ are primitive, i.e. $\overline{\boldsymbol{\Lambda}}\left(x_{i}\right)=0$. In other words, the algebra $(\mathcal{H}, \cdot)$ is freely generated by the primitive elements for the coproduct $\boldsymbol{\Lambda}$. In our case, we will exhibit a coproduct $\boldsymbol{\Delta}$ such that $(\mathcal{R}, \cdot, \boldsymbol{\Delta})$ satisfies $(\star)$ and, as a corollary of [LR06], we get that $(\mathcal{R}, \cdot)$ is freely generated by the primitive elements of $\boldsymbol{\Lambda}$

Definition 25. A primitive cut is a total cut of the form $([i],[p] \backslash[i])$ for some $0 \leq i \leq p$.

For example, the relation $\overbrace{2_{3}}$ admits a primitive cut at 2 . Every relation $\mathrm{T} \in \mathcal{R}_{p}$ admits at least two primitive cuts $(\varnothing,[p])$ and $([p], \varnothing)$ which we call the trivial primitive cuts. Moreover, $\mathrm{T}=\mathrm{R} \backslash \mathrm{S}$ if and only if $T$ admits a primitive cut at $|\mathrm{R}|$. In particular, if $T$ is indecomposable, then $T$ does not admit any non-trivial primitive cut. We define a coproduct $\boldsymbol{\Delta}$ on the basis $\mathbb{E} \mathcal{R}$ by

$$
\boldsymbol{\Delta}\left(\mathbb{E} \mathcal{R}^{\mathrm{T}}\right):=\sum_{T=\mathrm{R} \backslash \mathrm{S}} \mathbb{E} \mathcal{R}^{\mathrm{R}} \otimes \mathbb{E} \mathcal{R}^{\mathrm{S}}
$$


By definition, this is the dual of the product · on $\mathbb{E} \mathcal{R}$. This is also a sum over all primitive cuts of the relation $\mathrm{T}$ and by extension, $\overline{\mathbf{\Delta}}\left(\mathbb{E} \mathcal{R}^{\mathrm{T}}\right)$ is a sum over all non-trivial primitive cuts of $\mathrm{T}$.

Example 26. For instance

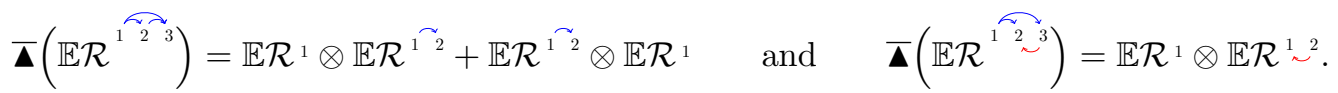

We have that $\mathbb{E} \mathcal{R}^{\mathrm{T}}$ is primitive for $\overline{\boldsymbol{\Lambda}}$ (i.e. $\overline{\mathbf{\Lambda}}\left(\mathbb{E} \mathcal{R}^{\mathrm{T}}\right)=0$ ) if and only if $\mathrm{T}$ is indecomposable. Now, Proposition 18 is a direct consequence of the following statement together with Theorem 24.

Proposition 27. $(\mathcal{R}, \cdot, \mathbf{\Lambda})$ is a unital infinitesimal bialgebra.

Proof. Let $\mathrm{R} \in \mathcal{R}_{m}$ and $\mathrm{S} \in \mathcal{R}_{n}$ with $p=m+n$. We have on the one hand

$$
A:=\overline{\mathbf{\Lambda}}\left(\mathbb{E} \mathcal{R}^{\mathrm{R}} \cdot \mathbb{E} \mathcal{R}^{\mathrm{S}}\right)=\overline{\mathbf{\Lambda}}\left(\mathbb{E} \mathcal{R}^{\mathrm{R} \backslash \mathrm{S}}\right)=\sum_{\mathrm{R}^{\prime} \backslash \mathrm{S}^{\prime}=\mathrm{R} \backslash \mathrm{S}} \mathbb{E} \mathcal{R}^{\mathrm{R}^{\prime}} \otimes \mathbb{E} \mathcal{R}^{\mathrm{S}^{\prime}},
$$

and on the other hand

$$
\begin{aligned}
B & :=\left(\mathbb{E} \mathcal{R}^{\mathrm{R}} \otimes \mathbb{E} \mathcal{R}^{\emptyset}\right) \cdot \overline{\boldsymbol{\Lambda}}\left(\mathbb{E} \mathcal{R}^{\mathrm{S}}\right)+\overline{\mathbf{\Lambda}}\left(\mathbb{E} \mathcal{R}^{\mathrm{R}}\right) \cdot\left(\mathbb{E} \mathcal{R}^{\emptyset} \otimes \mathbb{E} \mathcal{R}^{\mathrm{S}}\right)+\mathbb{E} \mathcal{R}^{\mathrm{R}} \otimes \mathbb{E} \mathcal{R}^{\mathrm{S}} \\
& =\sum_{\mathrm{S}=\mathrm{S}_{1} \backslash \mathrm{S}_{2}} \mathbb{E} \mathcal{R}^{\mathrm{R} \backslash \mathrm{S}_{1}} \otimes \mathbb{E} \mathcal{R}^{\mathrm{S}_{2}}+\sum_{\mathrm{R}=\mathrm{R}_{1} \backslash \mathrm{R}_{2}} \mathbb{E} \mathcal{R}^{\mathrm{R}_{1}} \otimes \mathbb{E} \mathcal{R}^{\mathrm{R}_{2} \backslash \mathrm{S}}+\mathbb{E} \mathcal{R}^{\mathrm{R}} \otimes \mathbb{E} \mathcal{R}^{\mathrm{S}} .
\end{aligned}
$$

We want to prove that $A=B$. The sum $A$ is over all non-trivial primitive cuts of $\mathrm{R} \backslash \mathrm{S}$. The relation $\mathrm{R} \backslash \mathrm{S}$ admits a primitive cut at $m$ by definition which means that the term $\mathbb{E} \mathcal{R}^{\mathrm{R}} \otimes \mathbb{E}^{\mathrm{S}}$ appears in the sum. Now, let $0<k<m$.

- If $\mathrm{R} \backslash \mathrm{S}$ admits a primitive cut at $k^{\prime}$, this means in particular that $\mathrm{R}$ admits a primitive cut at $k$. We have $\mathrm{R}=\mathrm{R}_{1} \backslash \mathrm{R}_{2}$ for some $\mathrm{R}_{1} \in \mathcal{R}_{k}$ and $\mathrm{R}_{2} \in \mathcal{R}_{m-k}$. It is easy to check that $\mathrm{R} \backslash \mathrm{S}$ restricted to $\{k+1, \ldots, p\}$ is indeed equal to $\mathrm{R}_{2} \backslash \mathrm{S}$.

- Reciprocally, if $\mathrm{R}$ admits a primitive cut at $k$, i.e. $\mathrm{R}=\mathrm{R}_{1} \backslash \mathrm{R}_{2}$ with $\mathrm{R}_{1} \in \mathcal{R}_{k}$, we have all $(i, j) \in \mathrm{R} \backslash \mathrm{S}$ and $(j, i) \notin \mathrm{R} \backslash \mathrm{S}$ for $i \leq k$ and $k<j \leq m$ by definition of the primitive cut and also for $i \leq k$ and $m<j \leq p$ by definition of $\mathrm{R} \backslash \mathrm{S}$. This means that $\mathrm{R} \backslash \mathrm{S}$ admits a primitive cut $\mathrm{R}^{\prime} \backslash S^{\prime}=R \backslash S$ at $k$. The relation $R^{\prime}$ is the restriction of $R \backslash S$ to $[k]$ and it is then equal to $R_{1}$. The relations $\mathrm{S}^{\prime}$ is the restriction of $\mathrm{R} \backslash \mathrm{S}$ to $\{k+1, \ldots, p\}$ and is equal to $\mathrm{R}_{2} \backslash \mathrm{S}$.

We can use a similar argument for $k>m$ and we then obtain that the primitive cuts of $\mathrm{R} \backslash \mathrm{S}$ exactly correspond to the primitive cuts of $\mathrm{S}$ which proves the result.

As an algebra, $\mathbf{k} \mathcal{R}$ is then generated by indecomposable relations. It is well known that there is a direct relation between the Hilbert series of an algebra and the generating series of its indecomposable elements. Namely, if

$$
F(x):=\sum_{n \geq 0} R_{n} x^{n}=1+x+4 x^{2}+64 x^{3}+4098 x^{4}+\ldots
$$

is the Hilbert series of $\mathbf{k} \mathcal{R}$, where $R_{n}=2^{n(n-1)}$ is the number of (reflexive) integer binary relations, then it is related to the the generating series $I(x)$ of indecomposable relations by

$$
\frac{1}{1-I(x)}=R(x)
$$

In particular, the number of indecomposable relations $I_{n}$ can be computed by an inclusionexclusion formula

$$
I_{n}=\sum_{n_{1}+\cdots+n_{k}=n}(-1)^{k+1} R_{n_{1}} \ldots R_{n_{k}}
$$

which gives the coefficients of Table 2. There does not seem to be another, more direct, combinatorial enumeration. Nevertheless, indecomposable relations do have an interesting structural property when looking at the weak order lattice.

Proposition 28. The set of indecomposable relations of size $n$ forms an upper-ideal of the weak order lattice on $\mathcal{R}_{n}$ (i.e. if $\mathrm{R}$ is indecomposable, then any $\mathrm{S}$ with $\mathrm{R} \preccurlyeq \mathrm{S}$ is also indecomposable). 


\begin{tabular}{r|lllll}
$n$ & 1 & 2 & 3 & 4 & 5 \\
\hline$R_{n}$ & 1 & 4 & 64 & 4096 & 1048576 \\
\hline$I_{n}$ & 1 & 3 & 57 & 3963 & 1040097
\end{tabular}

TABLE 2. Number of binary relations and indecomposable binary relations on $[n]$.

Proof. Consider two binary relations $\mathrm{R}, \mathrm{S} \in \mathcal{R}_{n}$ such that $\mathrm{R} \preccurlyeq \mathrm{S}$ and $\mathrm{S}$ admits a primitive cut at some $k$. For all $i \leq k<j$, we have $i \mathrm{~S} j$ and $j \not S i$ since $k$ is a primitive cut of $\mathrm{S}$. Since $\mathrm{R} \preccurlyeq \mathrm{S}$, we have $\mathrm{R}^{\operatorname{lnc}} \supseteq \mathrm{S}^{\operatorname{lnc}}$ and $\mathrm{R}^{\text {Dec }} \subseteq \mathrm{S}^{\text {Dec }}$, and thus $i \mathrm{R} j$ and $j \not \supset i$ for all $i \leq k<j$. This implies that $\mathrm{R}$ also admits a primitive cut at $k$.

Note however that the ideal of indecomposable relations might have multiple minimal elements. For example for $n=2$, there are 3 indecomposable relations (over 4 relations in total) and 2 minimal elements: 12 and $\underset{r_{2}}{\sim}$.

\section{INTEGER POSETS}

We now focus on integer posets, i.e. integer relations that are reflexive $(x \mathrm{R} x)$, transitive $(x \mathrm{R} y \mathrm{R} z \Rightarrow x \mathrm{R} z)$ and antisymmetric $(x \mathrm{R} y \Rightarrow y R x)$. Let $\mathcal{P}_{n}$ be the set of all posets on $[n]$ and let $\mathcal{P}:=\bigsqcup_{n>0} \mathcal{R}_{n}$.

As we will only work with posets in the rest of the paper, we generally prefer to use notations like $\triangleleft, \varangle, \dashv$ which speak for themselves, rather than our previous notations R, S for arbitrary binary relations. It also allows us to write $a \triangleright b$ for $b \triangleleft a$, in particular when $a<b$.

We still denote by $\preccurlyeq$ the weak order given in Definition 1 . The following statement is the keystone of [CPP17].

Theorem 29 ([CPP17, Thm. 1]). The weak order on the integer posets of $\mathcal{P}_{n}$ is a lattice.

We now define a Hopf algebra on posets. We consider the vector space $\mathbf{k} \mathcal{P}:=\bigoplus_{n \geq 0} \mathbf{k} \mathcal{P}_{n}$ indexed by all integer posets of arbitrary size. We denote by $(\mathbb{F} \mathcal{P} \triangleleft)_{\triangleleft \in \mathcal{P}}$ the standard basis of $\mathbf{k} \mathcal{P}$.

Proposition 30. For any $\mathrm{R}, \mathrm{S} \in \mathcal{R}$,

(i) if the shifted shuffle $\mathrm{R} \amalg \mathrm{S}$ contains at least a poset, then $\mathrm{R}$ and $\mathrm{S}$ are both posets,

(ii) if $\mathrm{R}$ and $\mathrm{S}$ are both posets, then all relations in the convolution $\mathrm{R} \star \mathrm{S}$ are posets.

Therefore, the vector subspace of $\mathbf{k} \mathcal{R}$ generated by integer relations which are not posets is a Hopf ideal of $(\mathbf{k} \mathcal{R}, \cdot, \triangle)$. The quotient of the integer relation algebra $(\mathbf{k} \mathcal{R}, \cdot, \triangle)$ by this ideal is thus a Hopf algebra $(\mathbf{k} \mathcal{P}, \cdot \triangle)$ on integer posets.

Proof. For (i), let $\mathrm{R} \in \mathcal{R}_{m}$ and $\mathrm{S} \in \mathcal{R}_{n}$ be such that the shifted shuffle $\mathrm{R} \Psi \mathrm{S}$ contains a poset $\mathrm{T}$. Then $\mathrm{R}=\mathrm{T}_{[m]}$ and $\mathrm{S}=\mathrm{T}_{\overline{[n]}}$ are antisymmetric and transitive since $\mathrm{T}$ is.

For (ii), consider two posets $\mathrm{R}, \mathrm{S} \in \mathcal{P}$ and let $\mathrm{T} \in \mathrm{R} \star \mathrm{S}$. Let $(X, Y)$ be the total cut of $\mathrm{T}$ such that $\mathrm{T}_{X}=\mathrm{R}$ and $\mathrm{T}_{Y}=\mathrm{S}$. We prove that $\mathrm{T}$ is a poset:

Antisymmetry: Let $u, v \in \mathbb{N}$. If $u$ and $v$ both belong to $X$ (resp. to $Y$ ), then $u$ T $v \Rightarrow v$ T $u$ since $\mathrm{T}_{X}=\mathrm{R}$ (resp. $\mathrm{T}_{Y}=\mathrm{S}$ ) is antisymmetric. Otherwise, $u \mathrm{~T} v$ if and only if $u \in X$ and $v \in Y$, while $v \mathrm{~T} u$ if and only if $v \in X$ and $u \in Y$. Thus $\mathrm{T}$ is antisymmetric.

Transitivity: Let $u, v, w \in \mathbb{N}$ such that $u \mathrm{~T} v \mathrm{~T} w$. If $u$ and $w$ both belong to $X$ (resp. to $Y$ ), then so does $v$ and $u \mathrm{~T} w$ since $\mathrm{T}_{X}=\mathrm{R}$ (resp. $\mathrm{T}_{Y}=\mathrm{S}$ ) is transitive. Otherwise, $u \in X$ and $w \in Y$ (since $\mathrm{T} \cap(Y \times X)=\varnothing$ and $u \mathrm{~T} v \mathrm{~T} w)$, thus $u \mathrm{~T} w($ since $X \times Y \subseteq \mathrm{T})$. Thus $\mathrm{T}$ is transitive.

Remark 31. Although not needed for the Hopf algebra quotient, observe that the convolution satisfies a property similar to Proposition 30 (i): if $\mathrm{R}, \mathrm{S} \in \mathcal{R}$ are such that the convolution $\mathrm{R} \star \mathrm{S}$ contains at least a poset, then $\mathrm{R}$ and $\mathrm{S}$ are both posets.

For any poset $\triangleleft$, we denote by $\mathbb{F} \mathcal{P} \triangleleft$ the image of $\mathbb{F} \mathcal{R}_{\triangleleft}$ through the trivial projection $\mathbf{k} \mathcal{R} \rightarrow \mathbf{k} \mathcal{P}$. 


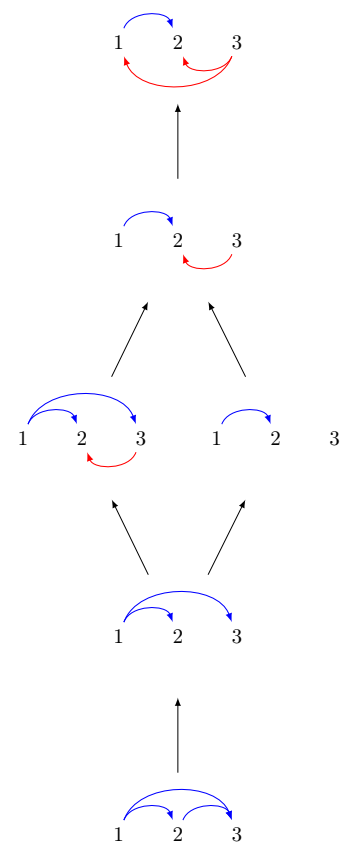

Figure 2. Interval corresponding to a product of posets. Its is obtained from Figure 1 by deleting all binary relations which are not posets.

Example 32. In practice, for two posets $\triangleleft, \triangleleft \in \mathcal{P}$, we compute the product $\mathbb{F} \mathcal{P} \triangleleft \cdot \mathbb{F} \mathcal{P} \triangleleft$ in $\mathbf{k} \mathcal{P}$ by deleting all non-poset summands in the product $\mathbb{F} \mathcal{R}_{\triangleleft} \cdot \mathbb{F} \mathcal{R}_{\triangleleft}$ in $\mathbf{k} \mathcal{R}$ :

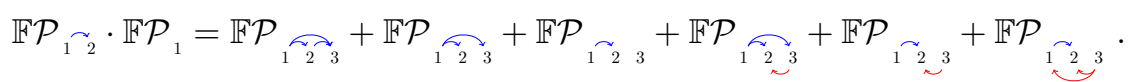

The coproduct is even simpler: all relations that appear in the coproduct $\triangle\left(\mathbb{F} \mathcal{R}_{\triangleleft}\right)$ of a poset $\triangleleft$ are automatically posets by Remark 31:

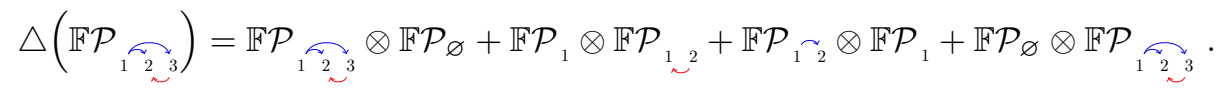

Proposition 33. For $\triangleleft \in \mathcal{P}_{m}$ and $\triangleleft \in \mathcal{P}_{n}$, the product $\mathbb{F} \mathcal{P}_{\triangleleft} \cdot \mathbb{F} \mathcal{P}_{\triangleleft}$ is the sum of $\mathbb{F} \mathcal{P}_{\dashv}$, where $\dashv$ runs over the interval between $\triangleleft \backslash \triangleleft$ and $\triangleleft / \triangleleft$ in the weak order on $\mathcal{P}_{m+n}$.

Proof. It is a direct consequence of Proposition 6 and the fact that for any two posets $\triangleleft, \varangle \in \mathcal{P}$, the relations $\triangleleft \backslash \triangleleft$ and $\triangleleft / \triangleleft$ are both posets.

Example 34. For instance, the product $\mathbb{F P}_{1_{2}} \cdot \mathbb{F P}_{1}$ corresponds to the interval of Figure 2

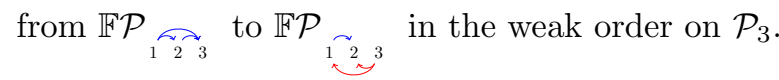

In a similar way, we directly obtain multiplicative bases $\mathbb{E} \mathcal{P}$ and $\mathbb{H} \mathcal{P}$ by taking the quotient of the bases $\mathbb{E} \mathcal{R}$ and $\mathbb{H} \mathcal{R}$ respectively:

$$
\mathbb{E} \mathcal{P}^{\triangleleft}=\sum_{\triangleleft \preccurlyeq \triangleleft} \mathbb{F} \mathcal{P} \triangleleft \quad \text { and } \quad \mathbb{H} \mathcal{P}^{\triangleleft}=\sum_{\triangleleft \preccurlyeq \triangleleft} \mathbb{F} \mathcal{P}_{\triangleleft}
$$

Note that if $\mathrm{R}$ is a relation that is not a poset, then the quotient $\mathbb{E} \mathcal{P}^{\mathrm{R}}$ of the element $\mathbb{E} \mathcal{R}^{\mathrm{R}}$ is not equal to 0 : the leading term $\mathbb{F}^{\mathrm{R}}$ is sent to 0 and so $\mathbb{E}^{\mathrm{R}}$ can be expressed as a sum of elements $\mathbb{E} \mathcal{P}^{R^{\prime}}$ where $R^{\prime}$ is a poset with $R^{\prime} \prec R$. 
Proposition 35. The sets $\left(\mathbb{E} \mathcal{P}^{\triangleleft}\right)_{\triangleleft \in \mathcal{P}}$ and $(\mathbb{H} \mathcal{P} \triangleleft)_{\triangleleft \in \mathcal{P}}$ form multiplicative bases of $\mathbf{k} \mathcal{P}$ with $\mathbb{E} \mathcal{P}^{\triangleleft} \cdot \mathbb{E} \mathcal{P}^{\triangleleft}=\mathbb{E} \mathcal{P}^{\triangleleft \backslash \triangleleft} \quad$ and $\quad \mathbb{H} \mathcal{P}^{\triangleleft} \cdot \mathbb{H} \mathcal{P} \triangleleft=\mathbb{H} \mathcal{P}^{\triangleleft / \triangleleft}$.

Besides, as an algebra, $\mathbf{k} \mathcal{P}$ is freely generated by the elements $(\mathbb{E} \mathcal{P} \triangleleft)$ where $\triangleleft$ is under-indecomposable and, equivalently, by the elements $(\mathbb{H} \mathcal{P} \triangleleft)$ where $\triangleleft$ is over-indecomposable.

Proof. This derives directly from Proposition 18 and the fact that if $\triangleleft$ and $\triangleleft$ are posets, then $\triangleleft \backslash \triangleleft$ and $\triangleleft / \triangleleft$ are also posets. To prove that the algebra is freely generated by the indecomposable elements, one can follow the proof of Proposition 22 as everything still holds when restricting to posets.

In the next two sections of the paper, we will use the Hopf algebra on integer posets constructed in this section to reinterpret classical Hopf algebras on permutations [MR95] (see Sections 3.1, 3.3.1 and 3.4.2), ordered partitions [Cha00] (see Section 3.3.3), binary trees [LR98] (see Sections 4.1 and 4.4) and Schröder trees [Cha00] (see Section 4.4). Moreover, we obtain Hopf structures on the intervals of the weak order (see Sections 3.3.2 and 3.4.1) and on the intervals of the Tamari lattice (see Section 4.4), that remained undiscovered to the best of our knowledge. All these algebras and their connections are summarized in Table 1 and Figure 3.

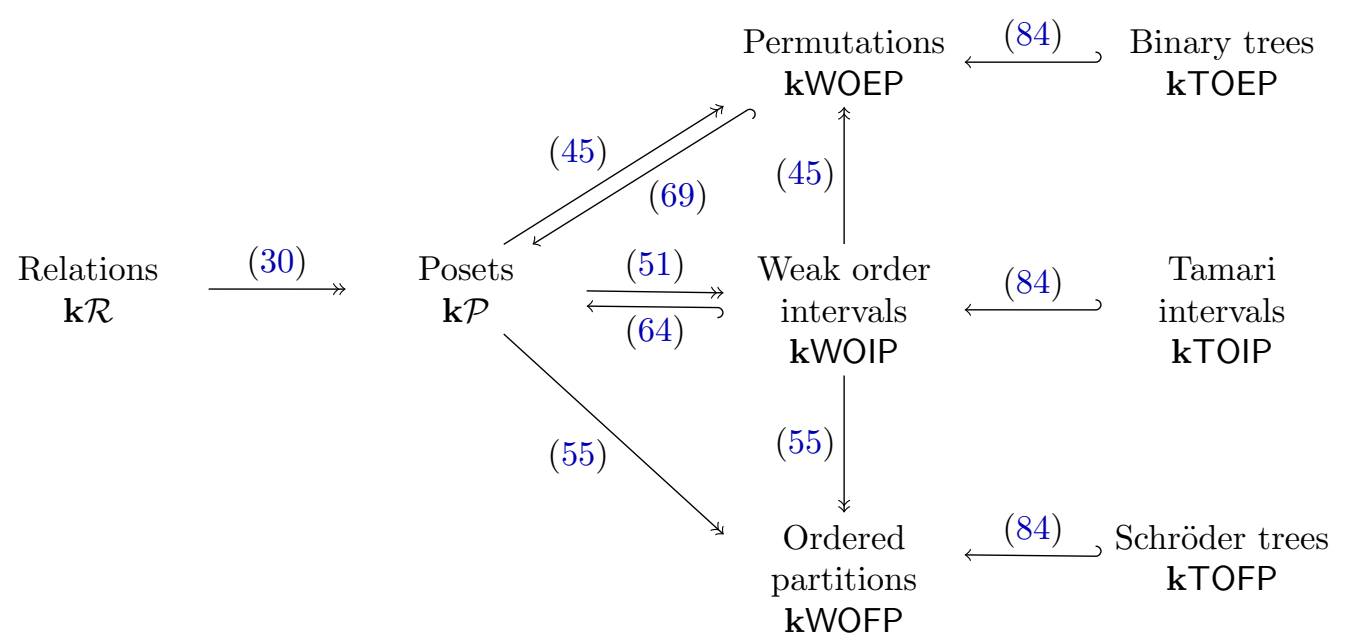

FiguRE 3. A roadmap through the different Hopf algebras studied in this paper. An arrow $\rightarrow$ indicates a quotient Hopf algebra, while an arrow $\hookrightarrow$ indicates a Hopf subalgebra. The label on each arrow refers to the corresponding proposition.

\section{Permutations, Weak order intervals, AND ORDERED PARTitions}

We now consider our first three families of specific integer posets. These families respectively correspond to the elements (WOEP), the intervals (WOIP) and the faces (WOFP) in the classical weak order on permutations. We construct Hopf algebras on WOEP, WOIP and WOFP as quotients (Section 3.3) or subalgebras (Section 3.4) of the integer poset Hopf algebra $(\mathcal{P}, \cdot, \triangle$ ). For the constructions as quotients, the important point is that all these families of posets are defined by local conditions on their relations, and that a contradiction to these conditions cannot be destroyed by the product or the coproduct. For the constructions as subalgebras, we use surjections from posets to WOEP or WOIP whose fibers are stable by product and coproduct. Using quotients or subalgebras, we construct Hopf algebras on WOEP (resp. WOFP) isomorphic to the Malvenuto-Reutenauer Hopf algebra on permutations [MR95] (resp. to the Chapoton Hopf algebra on surjections [Cha00]), and we obtain a Hopf algebra on intervals of the weak order that was not constructed earlier to the best of our knowledge. 
3.1. Permutations and the Malvenuto-Reutenauer algebra. Recall that the classical weak order on the permutations of $\mathfrak{S}_{n}$ is defined by $\sigma \preccurlyeq \tau$ if and only if $\operatorname{inv}(\sigma) \subseteq \operatorname{inv}(\tau)$, where $\operatorname{inv}(\sigma):=\left\{(a, b) \in[n]^{2} \mid a \leq b\right.$ and $\left.\sigma^{-1}(a) \leq \sigma^{-1}(b)\right\}$ denotes the inversion set of $\sigma$. This order is a lattice with minimal element $[1,2, \ldots, n]$ and maximal element $[n, \ldots, 2,1]$.

For two permutations $\sigma \in \mathfrak{S}_{m}$ and $\tau \in \mathfrak{S}_{n}$, the shifted shuffle $\sigma \bar{\Psi} \tau$ (resp. the convolution $\sigma \star \tau$ ) is the set of permutations of $\mathfrak{S}_{m+n}$ whose first $m$ values (resp. positions) are in the same relative order as $\sigma$ and whose last $n$ values (resp. positions) are in the same relative order as $\tau$. For example,

$$
\begin{aligned}
12 \varpi 231 & =\{12453,14253,14523,14532,41253,41523,41532,45123,45132,45312\}, \\
\text { and } \quad 12 \star 231 & =\{12453,13452,14352,15342,23451,24351,25341,34251,35241,45231\} .
\end{aligned}
$$

Recall that the Malvenuto-Reutenauer Hopf algebra [MR95] is the Hopf algebra on permutations with product $\cdot$ and coproduct $\triangle$ defined by

$$
\mathbb{F}_{\sigma} \cdot \mathbb{F}_{\tau}:=\sum_{\rho \in \sigma \varpi \tau} \mathbb{F}_{\rho} \quad \text { and } \quad \triangle\left(\mathbb{F}_{\rho}\right):=\sum_{\rho \in \sigma \star \tau} \mathbb{F}_{\sigma} \otimes \mathbb{F}_{\tau} .
$$

Example 36. For example, we have

$$
\mathbb{F}_{12} \cdot \mathbb{F}_{1}=\mathbb{F}_{123}+\mathbb{F}_{132}+\mathbb{F}_{312} \quad \text { and } \quad \triangle\left(\mathbb{F}_{132}\right)=\mathbb{F}_{132} \otimes \mathbb{F}_{\varnothing}+\mathbb{F}_{1} \otimes \mathbb{F}_{21}+\mathbb{F}_{12} \otimes \mathbb{F}_{1}+\mathbb{F}_{\varnothing} \otimes \mathbb{F}_{132}
$$

Remark 37. For $\sigma \in \mathfrak{S}_{m}$ and $\tau \in \mathfrak{S}_{n}$, we have $\sigma \bar{\tau} \tau=[\sigma \backslash \tau, \sigma / \tau]$. More generally, for any permutations $\sigma \preccurlyeq \sigma^{\prime} \in \mathfrak{S}_{m}$ and $\tau \preccurlyeq \tau^{\prime} \in \mathfrak{S}_{n}$, we have

$$
\left(\sum_{\sigma \preccurlyeq \lambda \preccurlyeq \sigma^{\prime}} \mathbb{F}_{\lambda}\right) \cdot\left(\sum_{\tau \preccurlyeq \mu \preccurlyeq \tau^{\prime}} \mathbb{F}_{\mu}\right)=\sum_{\sigma \backslash \tau \preccurlyeq \nu \preccurlyeq \sigma^{\prime} / \tau^{\prime}} \mathbb{F}_{\nu} .
$$

In other words, weak order intervals are stable by the product $\cdot$ on $\mathfrak{S}$. Note that there are not stable by the coproduct $\triangle$.

3.2. Weak order element, interval and face posets. We now briefly recall how the elements, the intervals and the faces of the classical weak order can be interpreted as specific interval posets as developed in [CPP17].

3.2.1. Elements. We interpret each permutation $\sigma \in \mathfrak{S}_{n}$ as the weak order element poset $\triangleleft_{\sigma}$ defined by $u \triangleleft_{\sigma} v$ if $\sigma^{-1}(u) \leq \sigma^{-1}(v)$. In other words, the poset $\triangleleft_{\sigma}$ is the chain $\sigma(1) \triangleleft_{\sigma} \ldots \triangleleft_{\sigma}$ $\sigma(n)$. See Figure 4 for an example with $\sigma=2751346$.

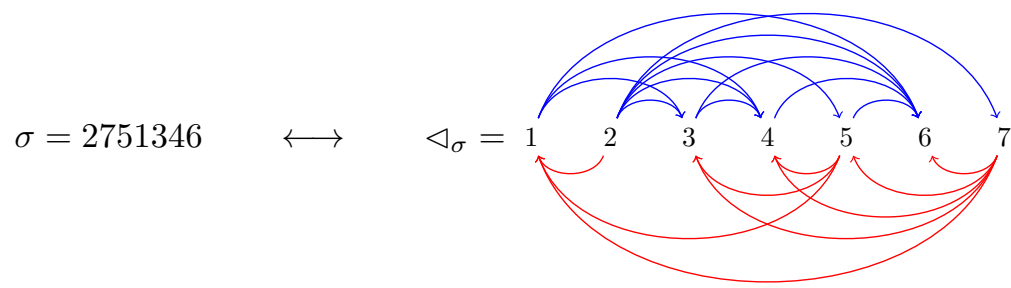

Figure 4. A Weak Order Element Poset (WOEP).

We define

$$
\operatorname{WOEP}_{n}:=\left\{\triangleleft_{\sigma} \mid \sigma \in \mathfrak{S}_{n}\right\} \quad \text { and } \quad \text { WOEP }:=\bigsqcup_{n \in \mathbb{N}} \text { WOEP }_{n} .
$$

These posets are clearly characterized as follows, which enables to recover the weak order on permutations.

Lemma 38. A poset $\triangleleft$ is in $\mathrm{WOEP}_{n}$ if and only if $a \triangleleft b$ or $a \triangleright b$ for all $a, b \in[n]$.

Proposition 39 ([CPP17, Prop. $23 \&$ 24]). The map $\sigma \mapsto \triangleleft_{\sigma}$ is a lattice isomorphism from the weak order on permutations of $\mathfrak{S}_{n}$ to the sublattice of the weak order on $\mathcal{P}_{n}$ induced by WOEP${ }_{n}$. 
3.2.2. Intervals. We now present a similar interpretation of the intervals of the weak order. For $\sigma \preccurlyeq \sigma^{\prime} \in \mathfrak{S}_{n}$, we consider the weak order interval $\left[\sigma, \sigma^{\prime}\right]:=\left\{\tau \in \mathfrak{S}_{n} \mid \sigma \preccurlyeq \tau \preccurlyeq \sigma^{\prime}\right\}$. The permutations of the interval $\left[\sigma, \sigma^{\prime}\right]$ are precisely the linear extensions of the weak order interval poset $\triangleleft_{\left[\sigma, \sigma^{\prime}\right]}:=\bigcap_{\sigma \preccurlyeq \tau \preccurlyeq \sigma^{\prime}} \triangleleft_{\tau}=\triangleleft_{\sigma} \cap \triangleleft_{\sigma^{\prime}}=\triangleleft_{\sigma^{\prime}}^{\text {Inc }} \cup \triangleleft_{\sigma}^{\text {Dec }}$, see the example on Figure 5 .

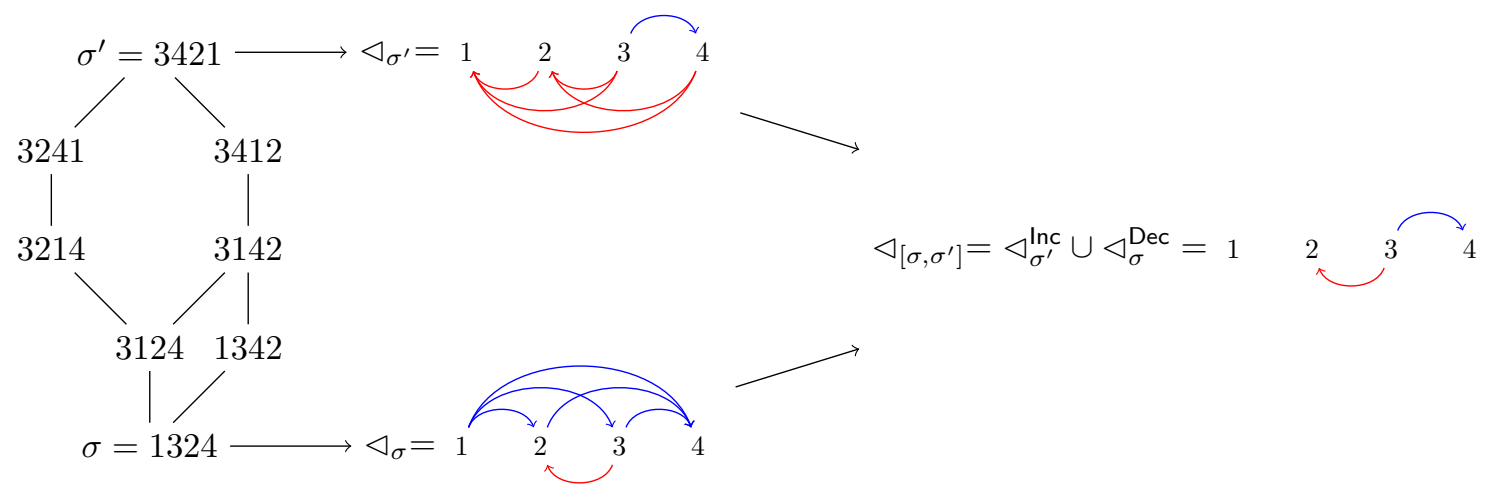

Figure 5. A Weak Order Interval Poset (WOIP).

We define

$$
\text { WOIP }_{n}:=\left\{\triangleleft_{\left[\sigma, \sigma^{\prime}\right]} \mid \sigma \preccurlyeq \sigma^{\prime} \in \mathfrak{S}_{n}\right\} \quad \text { and } \quad \text { WOIP }:=\bigsqcup_{n \in \mathbb{N}} \text { WOIP }_{n} .
$$

Weak order interval posets are precisely the integer posets which admit both a minimal and a maximal linear extension. They were characterized by A. Bjorner and M. Wachs [BW91] as follows.

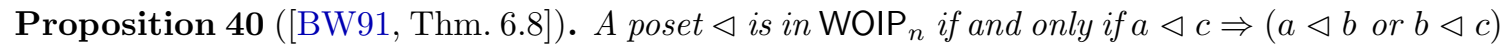
and $a \triangleright c \Rightarrow(a \triangleright b$ or $b \triangleright c)$ for all $1 \leq a<b<c \leq n$.

This condition clearly contains two separate conditions on the increasing subrelation and on the decreasing subrelation of $\triangleleft$, and it will be convenient to split these conditions. We thus consider the set IWOIP ${ }_{n}$ (resp. DWOIP $n$ ) of posets of $\mathcal{P}_{n}$ which admit a weak order maximal (resp. minimal) linear extension. These posets are characterized as follows.

Proposition 41 ([CPP17, Prop. 32]). For a poset $\triangleleft \in \mathcal{P}_{n}$,

- $\triangleleft \in \operatorname{IWOIP}_{n} \Longleftrightarrow \forall 1 \leq a<b<c \leq n, a \triangleleft c \Rightarrow a \triangleleft b$ or $b \triangleleft c$,

- $\triangleleft \in \mathrm{DWOIP}_{n} \Longleftrightarrow \forall 1 \leq a<b<c \leq n, a \triangleright c \Rightarrow a \triangleright b$ or $b \triangleright c$.

Moreover,

- if $\triangleleft \in \mathrm{IWOIP}_{n}$, its maximal linear extension is $\triangleleft^{\text {maxle }}:=\triangleleft \cup\{(b, a) \mid a<b$ incomparable in $\triangleleft\}$,

- if $\triangleleft \in \mathrm{DWOIP}_{n}$, its minimal linear extension is $\triangleleft^{\text {minle }}:=\triangleleft \cup\{(a, b) \mid a<b$ incomparable in $\triangleleft\}$.

See Figure 6 for an example.

Finally, the weak order on WOIP $_{n}$ corresponds to the Cartesian product lattice on the intervals, described in the following statement.

Proposition 42 ([CPP17, Prop. 27 \& Coro. 28]).

(i) If $\sigma \preccurlyeq \sigma^{\prime}$ and $\tau \preccurlyeq \tau^{\prime}$ in $\mathfrak{S}_{n}$, then $\triangleleft_{\left[\sigma, \sigma^{\prime}\right]} \preccurlyeq \triangleleft_{\left[\tau, \tau^{\prime}\right]} \Longleftrightarrow \sigma \preccurlyeq \tau$ and $\sigma^{\prime} \preccurlyeq \tau^{\prime}$.

(ii) The weak order on $\mathrm{WOIP}_{n}$ is a lattice with meet $\triangleleft_{\left[\sigma, \sigma^{\prime}\right]} \wedge_{\mathrm{WOIP}} \triangleleft_{\left[\tau, \tau^{\prime}\right]}=\triangleleft_{\left[\sigma \wedge_{\mathfrak{S}} \tau, \sigma^{\prime} \wedge_{\mathfrak{S}} \tau^{\prime}\right]}$ and join $\triangleleft_{\left[\sigma, \sigma^{\prime}\right]} \vee_{\text {WOIP }} \triangleleft_{\left[\tau, \tau^{\prime}\right]}=\triangleleft_{\left[\sigma \vee_{\mathfrak{S}} \tau, \sigma^{\prime} \vee_{\mathfrak{S}} \tau^{\prime}\right]}$. However, the weak order on WOIP Hs $_{n}$ not a sublattice of the weak order on $\mathcal{P}_{n}$. 


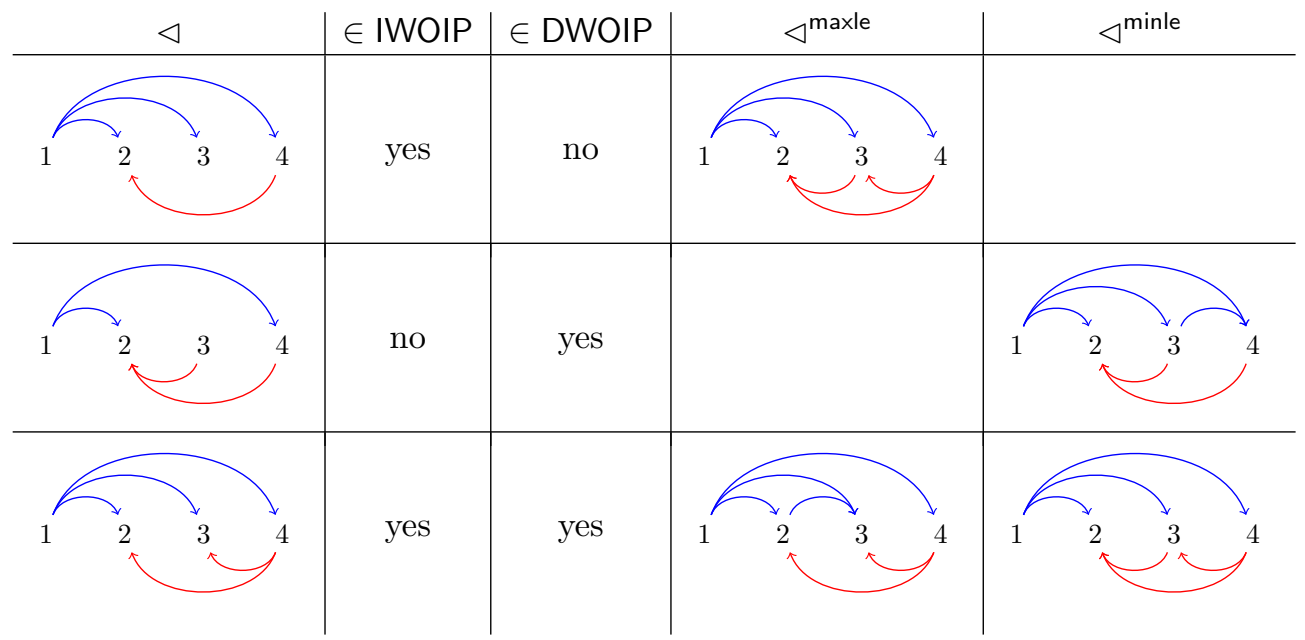

Figure 6. Examples of IWOIP and DWOIP with their maximum (resp. minimum) linear extensions.

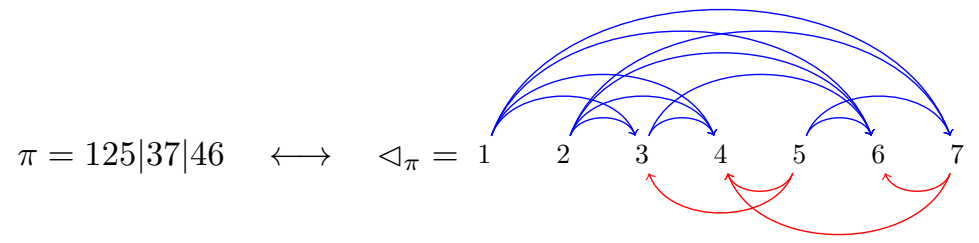

Figure 7. A Weak Order Face Poset (WOFP).

3.2.3. Faces. Recall that the permutahedron $\operatorname{Perm}(n):=\operatorname{conv}\left\{\left(\sigma_{1}, \ldots, \sigma_{n}\right) \mid \sigma \in \mathfrak{S}_{n}\right\}$ has vertices in bijections with permutations of $\mathfrak{S}_{n}$ and faces in bijections with ordered partitions of $[n]$. In this paper, we see an ordered partition $\pi$ of $[n]$ as a weak order face poset $\triangleleft_{\pi}$ defined by $u \triangleleft_{\pi} v$ if $\pi^{-1}(u)<\pi^{-1}(v)$ (meaning the block of $u$ is before the block of $v$ in $\pi$ ). See Figure 7 for an example with $\pi=125|37| 46$.

We define

$$
\text { WOFP }_{n}:=\left\{\triangleleft_{\pi} \mid \pi \text { ordered partition of }[n]\right\} \quad \text { and } \quad \text { WOFP }:=\bigsqcup_{n \in \mathbb{N}} \text { WOFP }_{n} .
$$

Again, the posets of WOFP $_{n}$ admit a simple local characterization and the weak order on WOFP $_{n}$ corresponds to a relevant lattice on faces of the permutahedron previously considered in $\left[\mathrm{KLN}^{+} 01\right.$, PR06, DHP18].

Proposition 43 ([CPP17, Prop. 30]). A poset $\triangleleft$ is in $\mathrm{WOFP}_{n}$ if and only if $\triangleleft \in \mathrm{WOIP}_{n}$ (Proposition 40) and $(a \triangleleft b \Longleftrightarrow b \triangleright c)$ and $(a \triangleright b \Longleftrightarrow b \triangleleft c)$ for all $a<b<c$ with $a \rtimes c$ and $a \not c$.

Proposition 44 ([CPP17, Sect. 2.1.3]).

(i) For any ordered partitions $\pi, \pi^{\prime}$ of $[n]$, we have $\triangleleft_{\pi} \preccurlyeq \triangleleft_{\pi^{\prime}} \Longleftrightarrow \pi \preccurlyeq \pi^{\prime}$ in the facial weak order of $\left[\mathrm{KLN}^{+} 01\right.$, PR06, DHP18].

(ii) The weak order on $\mathrm{WOFP}_{n}$ is a lattice but not a sublattice of the weak order on $\mathcal{P}_{n}$, nor on WOIP $_{n}$.

3.3. Quotient algebras. We now construct Hopf algebras on WOEP, WOIP and WOFP as quotient algebras of the integer poset algebra. For all these constructions, the crucial point is that all these families of posets are characterized by local conditions on their relations, and that a contradiction to these conditions cannot be destroyed by the product or the coproduct on posets. 
3.3.1. Elements. We first interpret the Malvenuto-Reutenauer Hopf algebra as a quotient of the integer poset Hopf algebra $(\mathbf{k} \mathcal{P}, \cdot, \triangle)$. For that, we use the characterization of Lemma 38.

Proposition 45. For any $\triangleleft, \triangleleft \in \mathcal{P}$,

(i) if the shifted shuffle $\triangleleft \bar{\square}$ contains a total order, then $\triangleleft$ and $\triangleleft$ are total orders,

(ii) if $\triangleleft$ and $\triangleleft$ are both total orders, then all relations in the convolution $\triangleleft \star \triangleleft$ are total orders. Therefore, the vector subspace of $\mathbf{k} \mathcal{P}$ generated by integer posets that are not total is a Hopf ideal of $(\mathbf{k} \mathcal{P}, \cdot, \triangle)$. The quotient of the integer poset Hopf algebra $(\mathbf{k} \mathcal{P}, \cdot, \triangle)$ by this ideal is thus a Hopf algebra $\left(\mathbf{k W O E P}{ }^{q u o}, \cdot, \triangle\right)$ on total orders.

Proof. For (i), let $\triangleleft \in \mathcal{P}_{m}$ and $\triangleleft \in \mathcal{P}_{n}$ be such that the shifted shuffle $\triangleleft \bar{\Psi}$ contains a total order $\dashv$. Then $\triangleleft=\dashv_{[m]}$ and $\triangleleft=\dashv \uparrow_{[n]}$ are total orders.

For (ii), consider two total orders $\triangleleft, \triangleleft \in$ WOEP and let $\dashv \in \triangleleft \star \triangleleft$. Let $(X, Y)$ be the total cut of $\dashv$ such that $\dashv_{X}=\triangleleft$ and $\dashv_{Y}=\varangle$. Let $u, v \in \mathbb{N}$. If $u$ and $v$ both belong to $X$ (resp. to $Y$ ), then $u \dashv v$ or $v \dashv u$ since $\dashv_{X}=\triangleleft$ (resp. $\left.\dashv_{Y}=\triangleleft\right)$ is total. Otherwise, $u \dashv v$ if and only if $u \in X$ and $v \in Y$, while $v \dashv u$ if and only if $v \in X$ and $u \in Y$. Thus $\dashv$ is total.

Remark 46. Although not needed for the Hopf algebra quotient, observe that the convolution satisfies a property similar to Proposition 45 (i): if $\triangleleft, \triangleleft \in \mathcal{P}$ are such that the convolution $\triangleleft \star \triangleleft$ contains at least a total order, then $\triangleleft$ and $\triangleleft$ are both total orders.

For any weak order element poset $\triangleleft$, we denote by $\mathbb{F W E} \triangleleft$ quo the image of $\mathbb{F} \mathcal{R}_{\triangleleft}$ through the trivial projection $\mathbf{k} \mathcal{R} \rightarrow \mathbf{k} \mathcal{P}$.

Example 47. In practice, for any two total orders $\triangleleft, \triangleleft \in$ WOEP, we compute the product $\mathbb{F W E}_{\triangleleft}^{\text {quo }} \cdot \mathbb{F W E}_{\triangleleft}^{\text {quo }}$ in kWOEPquo by deleting all summands not in WOEP in the product $\mathbb{F}_{\triangleleft} \cdot \mathbb{F} \mathcal{R} \triangleleft$ in $\mathbf{k} \mathcal{R}$ :

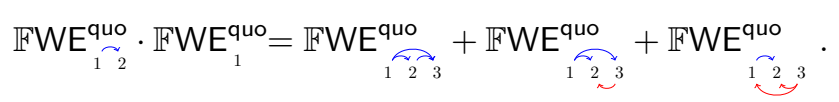

The coproduct is even simpler: all relations that appear in the coproduct $\triangle\left(\mathbb{F} \mathcal{R}_{\triangleleft}\right)$ of a total order $\triangleleft \in$ WOEP are automatically in WOEP by Remark 46 :

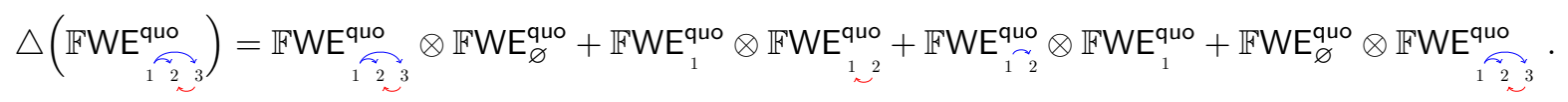

Proposition 48. The map $\mathbb{F}_{\sigma} \mapsto \mathbb{F W E}_{\triangleleft_{\sigma}}^{\text {quo }}$ defines a Hopf algebra isomorphism from the MalvenutoReutenauer Hopf algebra on permutations [MR95] to the quotient Hopf algebra $(\mathbf{k W O E P q u o}, \cdot, \triangle)$.

Proof. We just need to show that for any two permutations $\sigma \in \mathfrak{S}_{m}$ and $\tau \in \mathfrak{S}_{n}$, we have

$$
\left(\triangleleft_{\sigma} \bar{\Psi} \triangleleft_{\tau}\right) \cap \text { WOEP }=\left\{\triangleleft_{\rho} \mid \rho \in \sigma \bar{\Psi}\right\} \quad \text { and } \quad \triangleleft_{\sigma} \star \triangleleft_{\tau}=\left\{\triangleleft_{\rho} \mid \rho \in \sigma \star \tau\right\} .
$$

For the shuffle product, consider first an element of $\left(\triangleleft_{\sigma} \bar{\Psi} \triangleleft_{\tau}\right) \cap$ WOEP. By definition, it is of the form $\triangleleft_{\rho}$ for some $\rho \in \mathfrak{S}_{m+n}$. Moreover, $\triangleleft_{\sigma} \subset \triangleleft_{\rho}$ so that the first $m$ values of $\rho$ are in the order of $\sigma$ and $\overline{\triangleleft_{\tau}} \subset \triangleleft_{\rho}$ so that the last $n$ values of $\rho$ are in the order of $\tau$. Thus $\rho \in \sigma \bar{\Psi}$. Conversely, for $\rho \in \sigma \bar{\Psi}$, we have

$\triangleleft_{\rho}=\triangleleft_{\sigma} \cup \overline{\triangleleft_{\tau}} \cup\left\{(i, j) \mid i \in[m], j \in[n], \rho^{-1}(i)<\rho^{-1}(j)\right\} \cup\left\{(j, i) \mid i \in[m], j \in[n], \rho^{-1}(i)>\rho^{-1}(j)\right\}$.

Therefore, $\triangleleft_{\rho} \in \triangleleft_{\sigma} \bar{U} \triangleleft_{\tau}$. The proof for the convolution is similar and left to the reader.

Example 49. Compare Examples 36 and 47.

In particular, Proposition 37 can be restated on WOEP as follow.

Proposition 50. For any $\triangleleft \in \mathrm{WOEP}_{m}$ and $\triangleleft \in \mathrm{WOEP}_{n}$, the product $\mathbb{F W E q u o} \cdot \mathbb{F W E}_{\triangleleft}^{\text {quo }}$ is the sum of $\mathbb{F W E}_{\dashv}^{\text {quo }}$, where $\dashv$ runs over the interval between $\triangleleft \backslash \triangleleft$ and $\triangleleft / \triangleleft$ in the weak order on $\mathrm{WOEP}_{m+n}$.

Proof. It is a direct consequence of Proposition 6 and the fact that for any two $\triangleleft, \triangleleft \in$ WOEP, the relations $\triangleleft \backslash \triangleleft$ and $\triangleleft / \triangleleft$ are both total orders. Indeed, for any two permutations $\sigma$ and $\tau$, we have $\triangleleft_{\sigma} \backslash \triangleleft_{\tau}=\triangleleft_{\sigma \backslash \tau}$ and $\triangleleft_{\sigma} / \triangleleft_{\tau}=\triangleleft_{\sigma / \tau}$. 
3.3.2. Intervals. We now present a Hopf algebra structure on intervals of the weak order. Before we start, let us make some observations:

- By Remark 37, the product - of the Malvenuto-Reutenauer algebra provides a natural algebra structure on weak order intervals. However, this does not define a Hopf algebra on weak order intervals as intervals are not stable by the coproduct $\triangle$ on $\mathfrak{S}$. Indeed, note that $\triangle\left(\mathbb{F}_{1423}+\mathbb{F}_{4123}\right)$ contains $\mathbb{F}_{1} \otimes \mathbb{F}_{123}$ and $\mathbb{F}_{1} \otimes \mathbb{F}_{312}$ but not $\mathbb{F}_{1} \otimes \mathbb{F}_{132}$ while 132 belongs to the weak order interval $[123,312]$.

- To the best of our knowledge, the Hopf algebra presented below did not appear earlier in the literature. In fact, we are not aware of any Hopf algebra on weak order intervals.

- The construction below relies on the local conditions characterizing WOIP in Proposition 40. As shown in Proposition 41, these conditions can be split to characterize separately IWOIP and DWOIP. Therefore, we obtain similar Hopf algebra structures on IWOIP and DWOIP, although we do not explicitly state all results for IWOIP and DWOIP.

Proposition 51. For any $\triangleleft, \triangleleft \in \mathcal{P}$,

(i) if $(\triangleleft \bar{\Psi} \triangleleft) \cap$ WOIP $\neq \varnothing$, then $\triangleleft \in$ WOIP and $\triangleleft \in$ WOIP,

(ii) if $\triangleleft \in \mathrm{WOIP}$ and $\triangleleft \in \mathrm{WOIP}$, then $(\triangleleft \star \triangleleft) \subseteq$ WOIP.

Therefore, the vector subspace of $\mathbf{k} \mathcal{P}$ generated by $\mathcal{P} \backslash$ WOIP is a Hopf ideal of $(\mathbf{k} \mathcal{P}, \cdot, \triangle)$. The quotient of the integer poset algebra $(\mathbf{k} \mathcal{P}, \cdot, \triangle)$ by this ideal is thus a Hopf algebra $\left(\mathbf{k W O I P}{ }^{q u o}, \cdot, \triangle\right)$ on weak order intervals. A similar statement holds for IWOIP and DWOIP.

Proof. We make the proof for IWOIP, the proof for DWOIP is symmetric, and the result follows for WOIP $=$ IWOIP DDWOIP. For (i), let $\triangleleft \in \mathcal{P}_{m}$ and $\triangleleft \in \mathcal{P}_{n}$ be such that the shifted shuffle $\triangleleft \overline{ }$ contains a poset $\dashv \in \operatorname{IWOIP}_{m+n}$. Let $1 \leq a<b<c \leq m$ be such that $a \triangleleft c$. Then $a \dashv c$ (since $\dashv_{[m]}=\triangleleft$ ), which ensures that $a \dashv b$ or $b \dashv c$ (since $\dashv \in$ IWOIP $_{m+n}$ ), and we obtain that $a \triangleleft b$ or $b \triangleleft c$ (again since $\dashv_{[m]}=\triangleleft$ ). We conclude that $\triangleleft \in \mathrm{IWOIP}_{m}$ by the characterization of Proposition 41, and we prove similarly that $\triangleleft \in \mathrm{IWOIP}_{n}$.

For (ii), consider two weak order interval posets $\triangleleft, \triangleleft \in$ IWOIP and let $\dashv \in \triangleleft \star \triangleleft$. Let $(X, Y)$ be the total cut of $\dashv$ such that $\dashv_{X}=\triangleleft$ and $\dashv_{Y}=\triangleleft$. Consider $a<b<c$ such that $a \dashv c$. We distinguish three situations according to the repartition of $\{a, b, c\}$ in the partition $X \sqcup Y$ :

- Assume first that $\{a, b, c\} \subseteq X$. Since $\dashv_{X}=\triangleleft$ is in IWOIP, we obtain that $a \dashv b$ or $b \dashv c$. The argument is identical if $\{a, b, c\} \subseteq Y$.

- Assume now that $\{a, c\} \subseteq X$ and $b \in Y$. Then $a \dashv b$ since $X \times Y \subseteq \dashv$. The argument is identical if $\{a, c\} \in Y$ and $b \in X$.

- Finally, assume that $\{a, c\} \nsubseteq X$ and $\{a, c\} \nsubseteq \subseteq Y$. Then we have $a \in X$ and $c \in Y$ (since $a \dashv c$ and $(Y \times X) \cap \dashv=\varnothing)$. Since $X \times Y \subseteq \dashv$, we obtain that $a \dashv b$ if $b \in Y$, while $b \dashv c$ if $b \in X$.

We therefore obtain that $a \dashv c \Rightarrow(a \dashv b$ or $b \dashv c)$ and we conclude that $\dashv \in$ IWOIP by the characterization of Proposition 41.

Remark 52. Although not needed for the Hopf algebra quotient, observe that the convolution satisfies a property similar to Proposition 51 (i): if $\triangleleft, \triangleleft \in \mathcal{P}$ are such that the convolution $\triangleleft \star$ contains at least one element in WOIP, then $\triangleleft$ and $\triangleleft$ are both in WOIP.

For any weak order interval poset $\triangleleft$, we denote by $\mathbb{F W l} \mathrm{q}_{\triangleleft}^{\text {uo }}$ the image of $\mathbb{F} \mathcal{R}_{\triangleleft}$ through the trivial projection $\mathbf{k} \mathcal{R} \rightarrow \mathbf{k} \mathcal{P}$.

Example 53. In practice, for any two weak order interval posets $\triangleleft, \triangleleft \in$ WOIP, we compute the product $\mathbb{F W I} I_{\triangleleft}^{\text {quo }} \cdot \mathbb{F W} \mathrm{I}_{\triangleleft}^{\text {quo }}$ in $\mathrm{kWOIP}{ }^{\text {quo }}$ by deleting all summands not in WOIP in the product $\mathbb{F} \mathcal{R}_{\triangleleft} \cdot \mathbb{F} \mathcal{R}_{\triangleleft}$ in $\mathrm{k} \mathcal{R}$ :

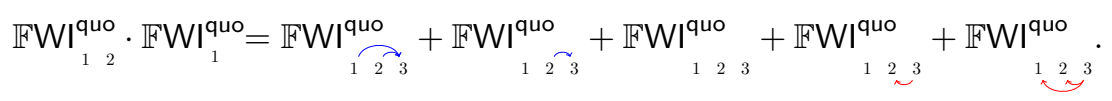


The coproduct is even simpler: all relations that appear in the coproduct $\triangle\left(\mathbb{F} \mathcal{R}_{\triangleleft}\right)$ of an element in WOIP are automatically in WOIP by Remark 52 :

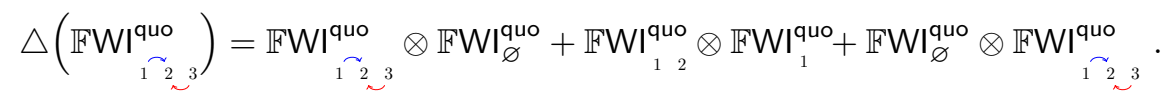

Proposition 54. For any $\triangleleft \in \mathrm{WOIP}_{m}$ and $\triangleleft \in \mathrm{WOIP}_{n}$, the product $\mathbb{F W I}_{\triangleleft}^{\text {quo }} \cdot \mathbb{F W l}_{\triangleleft}^{\text {quo }}$ is the sum of $\mathbb{F W I}_{-}^{\text {quo }}$, where $\dashv$ runs over the interval between $\triangleleft \backslash \triangleleft$ and $\triangleleft / \triangleleft$ in the weak order on $\mathrm{WOIP}_{m+n}$.

Proof. It is a direct consequence of Proposition 6 and the fact that for any two weak order interval posets $\triangleleft, \triangleleft \in$ WOIP, the relations $\triangleleft \backslash \triangleleft$ and $\triangleleft / \triangleleft$ are both weak order interval posets. Indeed, for any $\sigma \preccurlyeq \sigma^{\prime}$ and $\tau \preccurlyeq \tau^{\prime}$, we have $\triangleleft_{\left[\sigma, \sigma^{\prime}\right]} \backslash \triangleleft_{\left[\tau, \tau^{\prime}\right]}=\triangleleft_{\left[\sigma \backslash \tau, \sigma^{\prime} \backslash \tau^{\prime}\right]}$ and $\triangleleft_{\left[\sigma, \sigma^{\prime}\right]} / \triangleleft_{\left[\tau, \tau^{\prime}\right]}=\triangleleft_{\left[\sigma / \tau, \sigma^{\prime} / \tau^{\prime}\right]}$.

3.3.3. Faces. We now construct a Hopf algebra on faces of the permutahedra as a Hopf subalgebra of the poset Hopf algebra. We will see in Proposition 59 that the resulting Hopf algebra was already considered by F. Chapoton in [Cha00].

Proposition 55. For any $\triangleleft, \triangleleft \in \mathcal{P}$,

(i) if $(\triangleleft \bar{U} \triangleleft) \cap$ WOFP $\neq \varnothing$, then $\triangleleft \in$ WOFP and $\triangleleft \in$ WOFP,

(ii) if $\triangleleft \in$ WOFP and $\varangle \in$ WOFP, then $(\triangleleft \star \triangleleft) \subseteq$ WOFP.

Therefore, the vector subspace of $\mathbf{k} \mathcal{P}$ generated by $\mathcal{P} \backslash$ WOFP is a Hopf ideal of $(\mathbf{k} \mathcal{P}, \cdot, \triangle)$. The quotient of the poset Hopf algebra $(\mathbf{k} \mathcal{P}, \cdot, \triangle)$ by this ideal is thus a Hopf algebra $(\mathbf{k W O F P q u}, \cdot, \triangle)$ on faces of the permutahedron.

Proof. For (i), let $\triangleleft \in \mathcal{P}_{m}$ and $\triangleleft \in \mathcal{P}_{n}$ be such that the shifted shuffle $\triangleleft \bar{\Psi} \triangleleft$ contains a weak order face poset $\dashv \in$ WOFP $_{m+n}$. Since WOFP $\subset$ WOIP, Proposition 51 (i) ensures that $\triangleleft \in$ WOIP $_{m}$ and $\varangle \in$ WOIP $_{n}$. Consider now $1 \leq a<b<c \leq m$ such that $a \not c$ and $a \not c$. Then $a \rtimes c$ and $a \nvdash c$ (since $\left.\dashv_{[m]}=\triangleleft\right)$, which ensures that $a \dashv b \Longleftrightarrow b \vdash c$ and $a \vdash b \Longleftrightarrow b \dashv c$ (since $\dashv \in$ WOFP $_{m+n}$ ), and we obtain that $a \triangleleft b \Longleftrightarrow b \triangleright c$ and $a \triangleright b \Longleftrightarrow b \triangleleft c$ (again since $\left.\dashv_{[m]}=\triangleleft\right)$. We conclude that $\triangleleft \in$ WOFP $_{m}$ by the characterization of Proposition 43 , and we prove similarly that $\triangleleft \in$ WOFP $_{n}$.

For (ii), consider two weak order face posets $\triangleleft, \triangleleft \in$ WOFP and let $\dashv \in \triangleleft \star \triangleleft$. Since we have WOFP $\subset$ WOIP, Proposiiton 51 (ii) ensures that $\dashv \in$ WOIP. Let $(X, Y)$ be the total cut of $\dashv$ such that $\dashv_{X}=\triangleleft$ and $\dashv_{Y}=\triangleleft$. Consider $a<b<c$ such that $a \dashv c$ and $a \nvdash c$. Since $X \times Y \subseteq \dashv$, we obtain that $\{a, c\} \subseteq X$ or $\{a, c\} \subseteq Y$. Since $\dashv_{X}=\triangleleft$ and $\dashv_{Y}=\triangleleft$ are in WOFP, we obtain that $a \dashv b \Longleftrightarrow b \vdash c$ and $a \vdash b \Longleftrightarrow b \dashv c$. We conclude that $\dashv \in$ WOFP by the characterization of Proposition 43.

Remark 56. Although not needed for the Hopf algebra quotient, observe that the convolution satisfies a property similar to Proposition 55 (i): if $\triangleleft, \triangleleft \in \mathcal{P}$ are such that the convolution $\triangleleft \star \varangle$ contains at least one element in WOFP, then $\triangleleft$ and $\triangleleft$ are both in WOFP.

For any weak order face poset $\triangleleft$, we denote by $\mathbb{F W F} \triangleleft$ the image of $\mathbb{F} \mathcal{R}_{\triangleleft}$ through the trivial projection $\mathbf{k} \mathcal{R} \rightarrow \mathbf{k} \mathcal{P}$.

Example 57. In practice, for two weak order face posets $\triangleleft, \varangle \in$ WOFP, we compute the product $\mathbb{F W F}_{\triangleleft}^{\text {quo }} \cdot \mathbb{F} W^{\text {quo }}$ in kWOFPquo by deleting all summands not in WOFP in the product $\mathbb{F} \mathcal{R}_{\triangleleft} \cdot \mathbb{F} \mathcal{R}_{\triangleleft}$ in $\mathrm{k} \mathcal{R}$ :

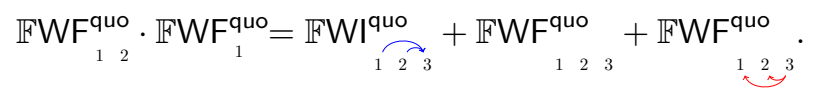

The coproduct is even simpler: all relations that appear in the coproduct $\triangle\left(\mathbb{F} \mathcal{R}_{\triangleleft}\right)$ of an element in WOFP are automatically in WOFP by Remark 56 :

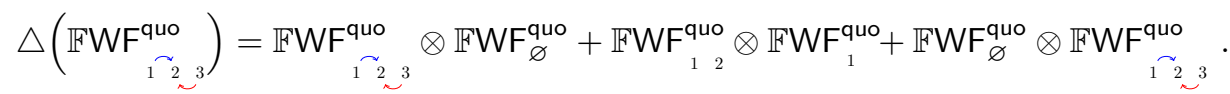

It turns out that the resulting algebra was studied in [Cha00]. Consider an ordered partition $\pi$ of $[m]$ into $k$ parts, and an ordered partition $\rho$ of $[n]$ into $\ell$ parts. As for permutations (see Section 3.1), the shifted shuffle $\pi \Psi \rho$ (resp. the convolution $\pi \star \rho$ ) is the set of ordered partitions 
whose first $k$ values (resp. first $m$ positions) are in the same relative order as $\pi$ and whose last $\ell$ values (resp. last $n$ positions) are in the same relative order as $\rho$. Here, relative order means in an earlier block, in the same block, or in a later block. Note that the shifted shuffle may merge blocks of $\pi$ with blocks of $\rho$ : all ordered partitions in $\pi \Psi \rho$ have at least $\min (k, \ell)$ blocks and at most $k+\ell$ blocks. In contrast, the convolution just adds up the numbers of blocks. For example,

$$
\begin{aligned}
& 1|2 \bar{\Psi} 2| 31=\{1|2| 4|53,1| 24|53,1| 4|2| 53,1|4| 253,1|4| 53|2,14| 2|53,14| 253, \\
& 14|53| 2,4|1| 2|53,4| 1|253,4| 1|53| 2,4|153| 2,4|53| 1 \mid 2\} \text {, } \\
& 1|2 \star 2| 31=\{1|2| 4|53,1| 3|4| 52,1|4| 3|52,1| 5|3| 42,2|3| 4 \mid 51, \\
& 2|4| 3|51,2| 5|3| 41,3|4| 2|51,3| 5|2| 41,4|5| 2 \mid 31\} .
\end{aligned}
$$

The Chapoton Hopf algebra [Cha00] is the Hopf algebra on ordered partitions with product · and coproduct $\triangle$ defined by

$$
\mathbb{F}_{\sigma} \cdot \mathbb{F}_{\tau}:=\sum_{\rho \in \sigma \varpi \tau} \mathbb{F}_{\rho} \quad \text { and } \quad \triangle\left(\mathbb{F}_{\rho}\right):=\sum_{\rho \in \sigma \star \tau} \mathbb{F}_{\sigma} \otimes \mathbb{F}_{\tau} .
$$

We refer to [Cha00, CP17] for more details and just provide an example of product and coproduct in this Hopf algebra.

Example 58. For example, we have

$$
\mathbb{F}_{12} \cdot \mathbb{F}_{1}=\mathbb{F}_{12 \mid 3}+\mathbb{F}_{123}+\mathbb{F}_{3 \mid 12} \quad \text { and } \quad \triangle\left(\mathbb{F}_{13 \mid 2}\right)=\mathbb{F}_{13 \mid 2} \otimes \mathbb{F}_{\varnothing}+\mathbb{F}_{12} \otimes \mathbb{F}_{1}+\mathbb{F}_{\varnothing} \otimes \mathbb{F}_{13 \mid 2} .
$$

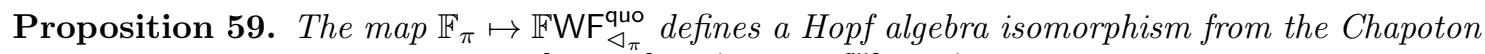
Hopf algebra on ordered partitions [Cha00] to $(\mathbf{k W O F P q u o}, \cdot, \triangle)$.

Proof. The proof is similar to that of Proposition 48 and left to the reader.

Example 60. Compare Examples 58 and 57.

Proposition 61. For any $\triangleleft \in \mathrm{WOFP}_{m}$ and $\triangleleft \in \mathrm{WOFP}_{n}$, the product $\mathbb{F W F}_{\triangleleft}^{\text {quo }} \cdot \mathbb{F W F}_{\triangleleft}^{\text {quo }}$ is the sum of $\mathbb{F W F}_{\dashv}^{\text {quo }}$, where $\dashv$ runs over the interval between $\triangleleft \backslash \triangleleft$ and $\triangleleft / \triangleleft$ in the weak order on $\mathrm{WOFP}_{m+n}$.

Proof. It is a direct consequence of Proposition 6 and the fact that for any two weak order face posets $\triangleleft, \varangle \in$ WOFP, the relations $\triangleleft \backslash \triangleleft$ and $\triangleleft / \triangleleft$ are both weak order face posets.

3.4. Subalgebras. We now construct Hopf algebras on WOEP and WOIP as subalgebras of the integer poset algebra. For this, we use surjections from all posets to WOEP or WOIP whose fibers are stable by product and coproduct. We consider the IWOIP increasing deletion, the DWOIP decreasing deletion, and the WOIP deletion defined in [CPP17, Sect. 2.1.4] by

$$
\begin{aligned}
\triangleleft^{\mathrm{IWOIPid}} & =\triangleleft \backslash\left\{(a, c) \mid \exists a<b_{1}<\cdots<b_{k}<c, a \not b_{1} \not \cdots \not b_{k} \not c\right\}, \\
\triangleleft^{\mathrm{DWOIPdd}} & =\triangleleft \backslash\left\{(c, a) \mid \exists a<b_{1}<\cdots<b_{k}<c, a \not b_{1} \not \cdots \not b_{k} \not c\right\}, \\
\triangleleft^{\text {WOIPd }} & =\left(\triangleleft^{\text {DWOIPdd }}\right)^{\text {IWOIPid }}=\left(\triangleleft^{\text {IWOIPid }}\right)^{\text {DWOIPdd }} .
\end{aligned}
$$

These operations are illustrated on Figure 8.

It is proved in [CPP17, Sect. 2.1.4] that $\triangleleft^{\text {IWOIPid }} \in$ IWOIP and $\triangleleft^{\text {DWOIPdd }} \in$ DWOIP for any $\triangleleft \in \mathcal{P}$. In fact, $\triangleleft \mapsto \triangleleft^{\text {IWOIPid }}$ (resp. $\triangleleft \mapsto \triangleleft^{\text {DWOIPdd }}$ ) is a projection from $\mathcal{P}$ to IWOIP (resp. DWOIP). Therefore, $\triangleleft^{\text {WOIPd }} \in$ WOIP for any $\triangleleft \in \mathcal{P}$ and $\triangleleft \mapsto \triangleleft^{\text {WOIPd }}$ is a projection from $\mathcal{P}$ to WOIP.

3.4.1. Intervals. Here, we use the fibers of the projections $\triangleleft \mapsto \triangleleft^{\text {IWOIPid }}, \triangleleft \mapsto \triangleleft^{\text {DWOIPdd }}$ and $\triangleleft \mapsto \triangleleft^{\text {WOIPd }}$ to construct Hopf subalgebras of the poset Hopf algebra. For this, we need the compatibility of these projections with the shuffle and the convolution on posets.

Lemma 62. For any $\triangleleft \in \mathcal{P}_{p}$ and any $1 \leq q \leq r \leq p$, we have

$$
\left(\triangleleft_{[q, r]}\right)^{\text {WOIPd }}=\left(\triangleleft^{\text {WOIPd }}\right)_{[q, r]} .
$$




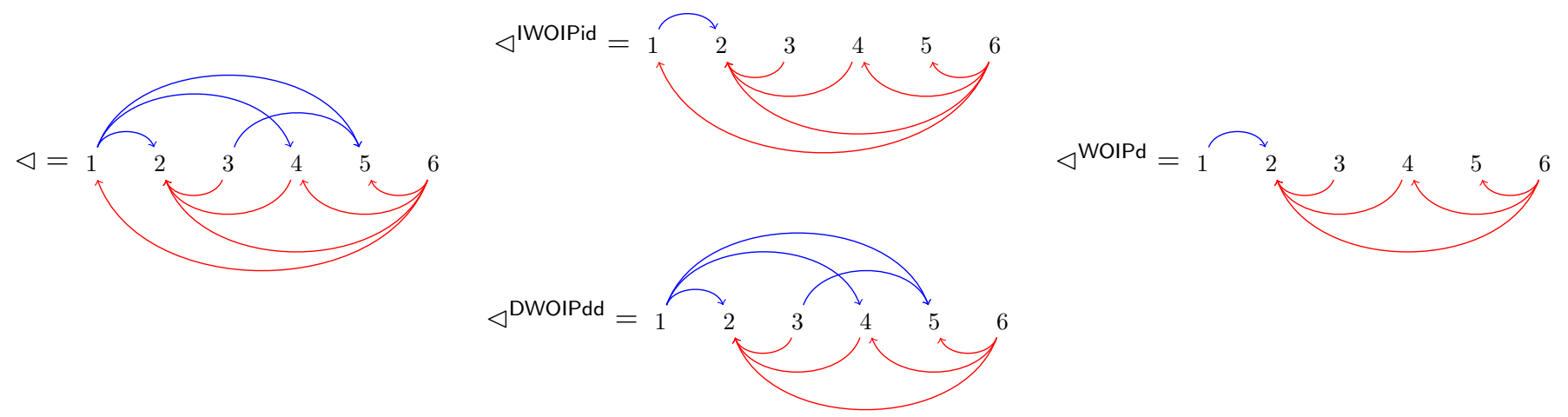

FIGURE 8. The IWOIP increasing deletion and the DWOIP decreasing deletion.

Therefore, for any $\triangleleft \in \mathrm{WOIP}_{m}$ and $\boldsymbol{\iota}^{\prime} \in \mathrm{WOIP}_{n}$,

$$
\left\{\triangleleft \in \mathcal{P}_{m+n} \mid\left(\triangleleft_{[m]}\right)^{\text {WOIPd }}=\triangleleft \text { and }\left(\triangleleft_{[n]}\right)^{\text {WOIPd }}=\triangleleft^{\prime}\right\}=\bigsqcup_{\prec \in \underset{\sim \text { WWOIP }}{\text { W. }}}\left\{\triangleleft \in \mathcal{P}_{m+n} \mid \triangleleft^{\text {WOIPd }}=\prec\right\} .
$$

A similar statement holds for $\triangleleft \mapsto \triangleleft^{\text {IWOIPid }}$ and for $\triangleleft \mapsto \triangleleft^{\text {DWOIPdd }}$.

Proof. We make the proof for IWOIP, the proof for DWOIP is symmetric, and the result follows for WOIP $=$ IWOIP $\cap$ DWOIP. The first statement immediately follows from the fact that the condition to delete $(a, c)$ in $\triangleleft^{\text {IWOIPid }}$ only involves relations of $\triangleleft$ in the interval $[a, c]$. By Proposition $6,\left(\triangleleft \bar{\Psi} \boldsymbol{\iota}^{\prime}\right) \cap$ IWOIP is the set of $\prec \in$ IWOIP $_{m+n}$ such that $\prec_{[m]}=\iota$ and $\prec \overline{[n]}=\varangle^{\prime}$, which shows the second statement.

Lemma 63. For any $\triangleleft \in \mathcal{P}_{p}$, the total cuts of $\triangleleft$ are precisely the total cuts of $\triangleleft^{\mathrm{WOIPd}}$. Moreover, if $(X, Y)$ is a total cut of $\triangleleft$, then

$$
\left(\triangleleft^{\text {WOIPd }}\right)_{X}=\left(\triangleleft_{X}\right)^{\text {WOIPd }} \quad \text { and } \quad\left(\triangleleft^{\text {WOIPd }}\right)_{Y}=\left(\triangleleft_{Y}\right)^{\text {WOIPd }} \text {. }
$$

Therefore, for any $\triangleleft \in$ WOIP $_{p}$ with a total cut $(X, Y)$,

$$
\left\{\triangleleft \in \mathcal{P}_{p} \mid \triangleleft^{\text {WOIPd }}=\triangleleft\right\}=\left\{\triangleleft \in \mathcal{P}_{p} \mid \begin{array}{ll}
\left(\triangleleft_{X}\right)^{\text {WOIPd }}=\triangleleft_{X} & \triangleleft \cap(Y \times X)=\varnothing \\
\left(\triangleleft_{Y}\right)^{\text {WOIPd }}=\triangleleft_{Y} & \text { and }(X \times Y) \subseteq \triangleleft
\end{array}\right\} .
$$

A similar statement holds for $\triangleleft \mapsto \triangleleft^{\text {IWOIPid }}$ and for $\triangleleft \mapsto \triangleleft^{\text {DWOIPdd }}$.

Proof. We make the proof for IWOIP, the proof for DWOIP is symmetric, and the result follows for WOIP $=$ IWOIP $\cap$ DWOIP. Consider a partition $[p]=X \sqcup Y$. Assume that $(X, Y)$ is a total cut of $\triangleleft$ and consider $x \in X$ and $y \in Y$. If $y<x$, then $x \triangleleft^{\text {IWOIPid }} y$ since $(x, y) \in \triangleleft^{\text {Dec }}=\left(\triangleleft^{\text {IWOIPid }}\right)^{\text {Dec }}$. If $x<y$, then $x \triangleleft^{\text {IWOIPid }} y$ since $x \triangleleft y$ and for any $x=b_{0}<b_{1}<\cdots<b_{k}<b_{k+1}=y$, we have $b_{\ell} \triangleleft b_{\ell+1}$, where $\ell$ is maximal such that $b_{\ell} \in X$. Finally, $y$ 丸lWOIPid $x$ since $y \Varangle x$ and $\triangleleft^{\text {IWOIPid }} \subseteq \triangleleft$. We conclude that $(X, Y)$ is a total cut of $\triangleleft^{\text {IWOIPid }}$. The reverse inclusion is similar.

We now consider a total cut $(X, Y)$ of $\triangleleft$ and prove that $\left(\triangleleft^{\text {IWOIPid }}\right)_{X}=\left(\triangleleft_{X}\right)^{\text {IWOIPid }}$ (the other equality is similar $)$. Observe first that $\left(\left(\triangleleft^{\text {IWOIPid }}\right)_{X}\right)^{\text {Dec }}=\left(\left(\triangleleft_{X}\right)^{\text {IWOIPid }}\right)^{\text {Dec }}=\triangleleft_{X}^{\text {Dec }}$ so we focus on increasing relations. Let $x_{1}<\cdots<x_{m}$ be the elements of $X$, and consider $1 \leq i<j \leq m$. If $(i, j) \in\left(\triangleleft^{\text {IWOIPid }}\right)_{X}$, then $x_{i} \triangleleft x_{j}$ and there is no $x_{i}<b_{1}<\cdots<b_{k}<x_{j}$ such that $x_{i} \Varangle b_{1} \Varangle$ $\cdots \rtimes b_{k} \rtimes x_{j}$. In particular, there is no such $b_{1}, \ldots, b_{k}$ in $X$ and we obtain that $(i, j) \in\left(\triangleleft_{X}\right)^{\text {IWOIPid }}$. Conversely, assume that $(i, j) \in\left(\triangleleft_{X}\right)^{\text {IWOIPid }}$ and consider $x_{i}=b_{0}<b_{1}<\cdots<b_{k}<b_{k+1}=x_{j}$. We distinguish two cases:

- If $b_{1}=x_{i_{1}}, \ldots, b_{k}=x_{i_{k}}$ all belong to $X$. Since $(i, j) \in\left(\triangleleft_{X}\right)^{\text {IWOIPid }}$, there is $\ell$ such that $i_{\ell} \triangleleft_{X} i_{\ell+1}$. This implies that $b_{\ell} \triangleleft b_{\ell+1}$.

- Otherwise, consider the last $\ell$ such that $b_{\ell} \in X$. Then $b_{\ell+1} \in Y$ and we have $b_{\ell} \triangleleft b_{\ell+1}$. 
In both cases, we have obtained that there is $\ell$ such that $b_{\ell} \triangleleft b_{\ell+1}$. We therefore obtain that $\left(x_{i}, x_{j}\right) \in \triangleleft^{\text {IWOIPid }}$, so that $(i, j) \in\left(\triangleleft_{X}\right)^{\text {IWOIPid }}$.

Finally, the last statement is just a reformulation of the former.

For $\triangleleft \in$ WOIP, consider the element

$$
\mathbb{F W} I_{\triangleleft}^{\text {sub }}:=\sum \mathbb{F} \mathcal{P}_{\triangleleft}
$$

where the sum runs over all $\triangleleft \in \mathcal{P}$ such that $\triangleleft^{\text {WOIPd }}=\triangleleft$. We denote by $\mathbf{k W O I P}^{\text {sub }}$ the linear subspace of $\mathbf{k} \mathcal{P}$ spanned by the elements $\left.\mathbb{F W}\right|^{\text {sub }}$ for $\varangle \in$ WOIP. We define similarly subspaces spanned by the fibers of the IWOIP and the DWOIP deletions.

Proposition 64. The subspace $\mathbf{k W O I P}^{\text {sub }}$ is stable by the product $\cdot$ and the coproduct $\triangle$ and thus defines a Hopf subalgebra of $(\mathbf{k} \mathcal{P}, \cdot \triangle)$. A similar statement holds for IWOIP and DWOIP.

Proof. The proof is a computation relying on Lemmas 62 and 63. We therefore make the proof here for WOIP, the same proof works verbatim for IWOIP or DWOIP. We first show the stability by product. For $\varangle \in$ WOIP $_{m}$ and $\varangle^{\prime} \in$ WOIP $_{n}$, we have by Lemma 62

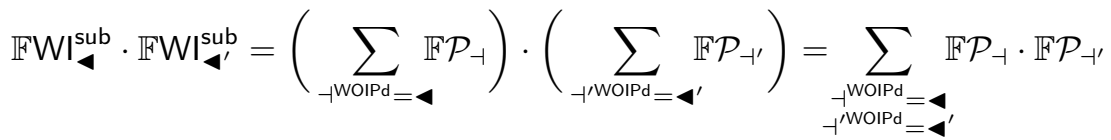

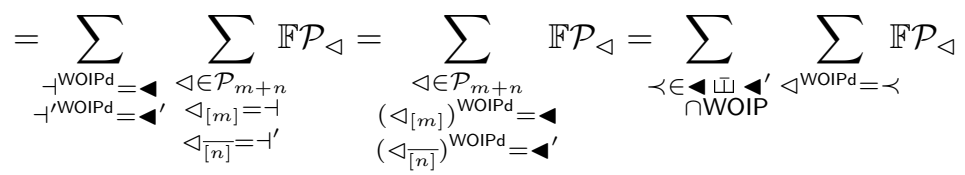

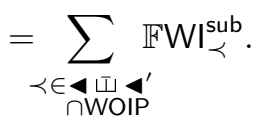

We now show the stability by coproduct. For $\boldsymbol{\iota} \in \mathrm{WOIP}_{p}$, we have by Lemma 63

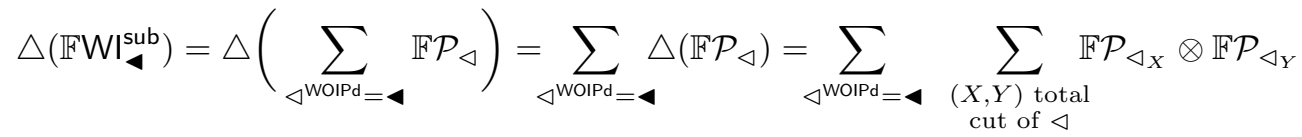

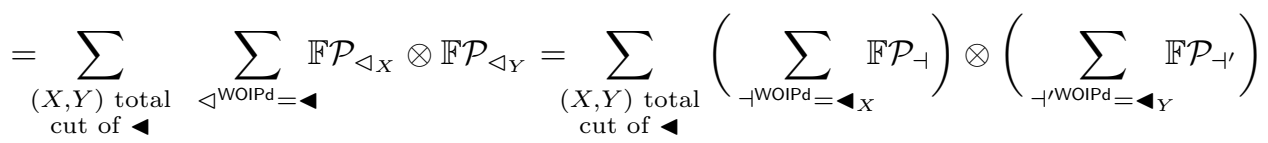

$$
\begin{aligned}
& =\sum_{\substack{(X, Y) \text { total } \\
\text { cut of } \triangleleft}} \mathbb{F W l}_{\iota_{X}}^{\text {sub }} \otimes \mathbb{F W I}_{\triangleleft_{Y}}^{\text {sub }}
\end{aligned}
$$

Proposition 65. The map $\mathbb{F W I}_{4}^{\text {quo }} \mapsto \mathbb{F W I}_{4}^{\text {sub }}$ defines a Hopf algebra isomorphism from the quotient Hopf algebra kWOIPqu to the Hopf subalgebra kWOIP ${ }^{\text {sub }}$. A similar statement holds for IWOIP and DWOIP.

Proof. This is immediate since the formulas for the product and coproduct on the bases (FWIquo ) and $\left(\mathbb{F W I}_{\boldsymbol{\triangleleft}}^{\text {sub }}\right)$ coincide:

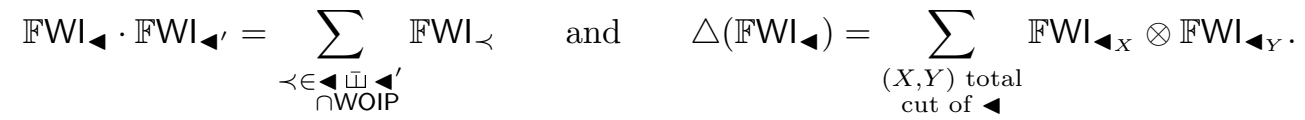

3.4.2. Elements. We now construct a Hopf algebra on WOEP as a subalgebra of the integer poset algebra. To this end, consider the two maps from $\mathcal{P}$ to WOEP defined by

$$
\triangleleft^{\text {WOEPid }}:=\left(\triangleleft^{\text {IWOIPid }}\right)^{\text {maxle }} \quad \text { and } \quad \triangleleft^{\text {WOEPdd }}:=\left(\triangleleft^{\text {DWOIPdd }}\right)^{\text {minle }} .
$$

We need to describe the fibers of these maps. 
Lemma 66. For $\triangleleft \in \mathcal{P}_{n}$ and $\triangleleft \in \mathrm{WOEP}_{n}$, we have

- $\triangleleft^{\mathrm{WOEPid}}=\triangleleft \Longleftrightarrow$ for all $(a, c) \in(\triangleleft \backslash \triangleleft)^{\operatorname{Inc}}$ there exists $a<b<c$ such that $a \triangleright b \triangleright c$,

- $\triangleleft^{\text {WOEPdd }}=\triangleleft \Longleftrightarrow$ for all $(c, a) \in(\triangleleft \backslash \triangleleft)^{\text {Dec }}$ there exists $a<b<c$ such that $a \triangleleft b \triangleleft c$.

Proof. We only prove the first statement, the second is symmetric. Assume $\triangleleft$ WOEPid $=4$ and $(a, c) \in(\triangleleft \backslash \triangleleft)^{\mathrm{Inc}}$. Since $(a, c)$ is deleted in $\triangleleft^{\text {WOEPid }}=\left(\triangleleft^{\text {IWOIPid }}\right)^{\text {maxle }}$, it is already deleted in $\triangleleft^{\text {IWOIPid }}$. Therefore, there exists $a<b_{1}<\cdots<b_{k}<c$ such that $a \Varangle b_{1} \nless \cdots \nless b_{k} \Varangle c$. By definition of $\triangleleft=\left(\triangleleft^{\text {IWOIPid }}\right)^{\text {maxle }}$, this implies that $a>b_{1} \triangleright \ldots b_{k} \triangleright c$.

Conversely, assume that for all $(a, c) \in(\triangleleft \backslash \triangleleft)^{\ln c}$ there exists $a<b<c$ such that $a \triangleright b \downarrow c$. Assume by contradiction that $\triangleleft^{\text {WOEPid }} \neq \triangleleft$. By definition of $\triangleleft^{\text {WOEPid }}=\left(\triangleleft^{\text {IWOIPid }}\right)^{\text {maxle }}$, this implies that there exists $(a, c) \in\left(\triangleleft^{\text {IWOIPid }} \backslash \triangleleft\right)^{\text {Inc }}$. Choose such an $(a, c)$ with $c-a$ minimal. Since $(a, c) \in\left(\triangleleft^{\text {IWOIPid }} \backslash \triangleleft\right)^{\operatorname{lnc}} \subseteq(\triangleleft \backslash \triangleleft)^{\text {Inc }}$, there exists $a<b<c$ such that $a \triangleright b \triangleright c$. Since $a \triangleleft^{\text {IWOIPid }} c$, we have $a \triangleleft^{\text {IWOIPid }} b$ or $b \triangleleft^{\text {IWOIPid }} c$, thus either $(a, b)$ or $(b, c)$ belongs to $\left(\triangleleft^{\text {IWOIPid }} \backslash \triangleleft\right)^{\text {Inc }}$ contradicting the minimality of $c-a$.

We now study the compatibility of the projections $\triangleleft \mapsto \triangleleft^{\text {WOEPid }}$ and $\triangleleft \mapsto \triangleleft$ WOEPdd with poset restrictions and cuts.

Lemma 67. For any $\triangleleft \in \mathcal{P}_{p}$ and any $1 \leq q \leq r \leq p$, we have

$$
\left(\triangleleft_{[q, r]}\right)^{\text {WOEPid }}=\left(\triangleleft^{\text {WOEPid }}\right)_{[q, r]} \quad \text { and } \quad\left(\triangleleft_{[q, r]}\right)^{\text {WOEPdd }}=\left(\triangleleft^{\text {WOEPdd }}\right)_{[q, r]} .
$$

Proof. Same as Lemma 62 since the presence of $(a, c)$ in both $\triangleleft \mapsto \triangleleft^{\text {IWOIPid }}$ and $\triangleleft \mapsto \triangleleft^{\text {maxle }}$ only depends on the relations of $\triangleleft$ in the interval $[a, c]$.

In contrast, Lemma 63 does not apply verbatim here since $\triangleleft$ has in general less total cuts than $\triangleleft^{\text {WOEPid }}$ and $\triangleleft^{\text {WOEPdd }}$. We adapt this lemma as follows.

Lemma 68. For any $\triangleleft \in \mathcal{P}_{p}$, all total cuts of $\triangleleft$ are total cuts of both $\triangleleft$ WOEPid and $\triangleleft$ WOEPdd. Moreover, for any $\triangleleft \in \mathcal{P}_{p}$ and $\triangleleft \in \operatorname{WOEP}_{p}$, and for any total cut $(X, Y)$ of both $\triangleleft$ and $\triangleleft$,

- $\triangleleft^{\text {WOEPid }}=\triangleleft$ if and only if $\left(\triangleleft_{X}\right)^{\text {WOEPid }}=\triangleleft_{X}$ and $\left(\triangleleft_{Y}\right)^{\text {WOEPid }}=\triangleleft_{Y}$,

- $\triangleleft^{\text {WOEPdd }}=\triangleleft$ if and only if $\left(\triangleleft_{X}\right)^{\text {WOEPdd }}=\triangleleft_{X}$ and $\left(\triangleleft_{Y}\right)^{\text {WOEPdd }}=\varangle_{Y}$,

Proof. We only prove the statement for $\triangleleft^{\text {WOEPid }}$, the proof for $\triangleleft^{\text {WOEPdd }}$ is symmetric. Consider a total cut $(X, Y)$ of $\triangleleft$ and let $x \in X$ and $y \in Y$. If $x>y$, then $(x, y) \in \triangleleft^{\text {Dec }} \subseteq \triangleleft^{\text {WOEPid }}$. If $x<y$, then for any $x<b<y$, we have either $b \in X$ and thus $b \triangleleft y$, or $b \in Y$ and thus $x \triangleleft b$. Therefore, $(x, y) \in \triangleleft^{\text {IWOIPid }} \subseteq \triangleleft^{\text {WOEPid }}$. We conclude that $(X, Y)$ is also a total cut of $\triangleleft$ WOEPid.

Consider now $\triangleleft \in \mathcal{P}_{p}$ and $\varangle \in$ WOEP $_{p}$, and a total cut $(X, Y)$ of both $\triangleleft$ and $\varangle$. Assume first that $\triangleleft^{\text {WOEPid }}=\triangleleft$. Consider $(a, c) \in\left(\triangleleft_{X} \backslash \triangleleft_{X}\right)^{\text {Inc }}$. Since $\triangleleft^{\text {WOEPid }}=\varangle$ and $(a, c) \in(\triangleleft \backslash \triangleleft)$, Lemma 66 ensures that there is $a<b<c$ such that $a>b>c$. Since $(X, Y)$ is a cut of 4 , we obtain that $b \in X$. Therefore, $a \boldsymbol{\nabla}_{X} b \boldsymbol{\nabla}_{X} c$. We conclude by Lemma 66 that $\left(\triangleleft_{X}\right)^{\text {WOEPid }}=\mathbf{\triangleleft}_{X}$. The proof is identical for $\left(\triangleleft_{Y}\right)^{\text {WOEPid }}=\triangleleft_{Y}$.

Conversely, assume that $\left(\triangleleft_{X}\right)^{\text {WOEPid }}=\varangle_{X}$ and $\left(\triangleleft_{Y}\right)^{\text {WOEPid }}=\varangle_{Y}$. Consider $(a, c) \in(\triangleleft \backslash \triangleleft)^{\operatorname{lnc}}$. Since $(X, Y)$ is a total cut of both $\triangleleft$ and $\triangleleft$, we know that $a$ and $c$ either both belong to $X$ or both belong to $Y$, say for instance $X$. Since $\left(\triangleleft_{X}\right)^{\text {WOEPid }}=\triangleleft_{X}$ we now that there exists $a<b<c$ such that $a \nabla_{X} b \nabla_{X} c$. We conclude by Lemma 66 that $\triangleleft^{\text {WOEPid }}=\mathbf{4}$.

For $\triangleleft \in$ WOEP, consider the elements

$$
\mathbb{F W E}_{\triangleleft}^{\text {subi }}:=\sum_{\substack{\triangleleft \in \mathcal{P} \\ \triangleleft \text { WOEPid }=\triangleleft}} \mathbb{F} \mathcal{P}_{\triangleleft} \text { and } \mathbb{F W E} E_{\triangleleft}^{\text {subd }}:=\sum_{\substack{\triangleleft \in \mathcal{P} \\ \triangleleft \text { WOEPdd }}} \mathbb{F} \mathcal{P}_{\triangleleft} .
$$

We denote by $\mathbf{k W O E P}{ }^{\text {subi }}$ and $\mathbf{k W O E P ^ { \text { subd } }}$ the linear subspaces of $\mathbf{k} \mathcal{P}$ spanned by the elements $\mathbb{F W E} \mathbf{4}^{\text {subi }}$ and $\mathbb{F W E}_{\triangleleft}^{\text {subd }}$ respectively for $\iota \in$ WOEP.

Proposition 69. The subspaces $\mathrm{kWOEP}^{\text {subi }}$ and $\mathrm{kWOEP}^{\mathrm{subd}}$ are stable by the product $\cdot$ and the coproduct $\triangle$ and thus defines Hopf subalgebras of $(\mathbf{k} \mathcal{P}, \cdot, \triangle)$. 
Proof. We only make the proof for kWOEP ${ }^{\text {subi }}$, the statement for kWOEP ${ }^{\text {subd }}$ is symmetric. We first show the stability by product. Using Lemma 67 , and the exact same computation as in the first part of the proof of Proposition 64, replacing WOIP by WOEP, we obtain that

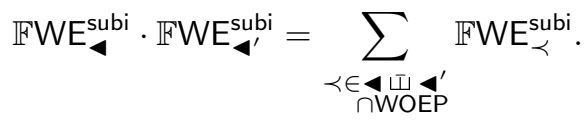

We now show the stability by coproduct. For $\triangleleft \in \mathrm{WOEP}_{p}$, we have by Lemma 68

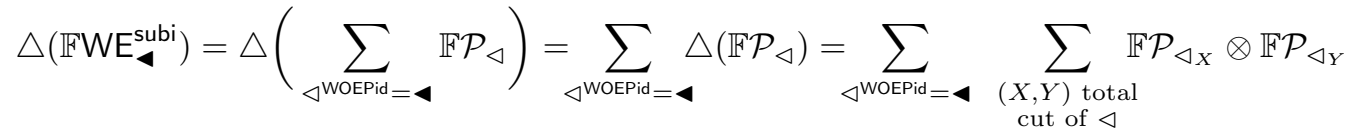

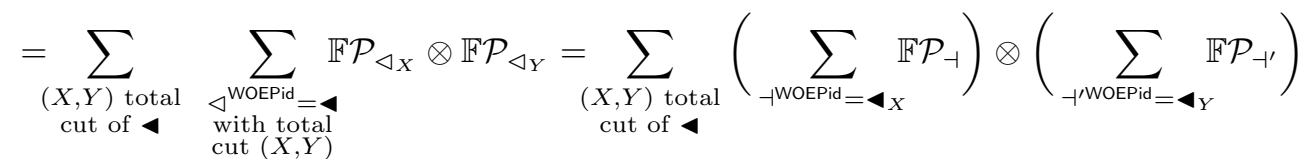

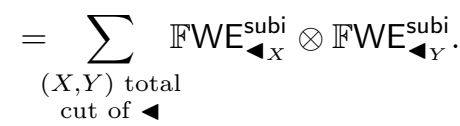

Proposition 70. The map $\mathbb{F W E} 4 \mapsto \mathbb{F W}$ subi (resp. $\mathbb{F W E}$ quo $\mapsto \mathbb{F W}$ subd $)$ defines a Hopf algebra isomorphism from the quotient Hopf algebra kWOEPqu to the Hopf subalgebra kWOEP ${ }^{\text {subi }}$ (resp. kWOEP ${ }^{\text {subd }}$ ). Therefore, $\mathrm{kWOEP}^{\text {subi }}$ and $\mathrm{kWOEP}^{\text {subi }}$ are isomorphic to the MalvenutoReutenauer Hopf algebra on permutations [MR95].

Proof. This is immediate since the formulas for the product and coproduct on the bases (FWEquo), $\left(\mathbb{F W E} E^{\text {subd }}\right)$ and $\left(\mathbb{F W E}_{\triangleleft}^{\text {subd }}\right)$ coincide:

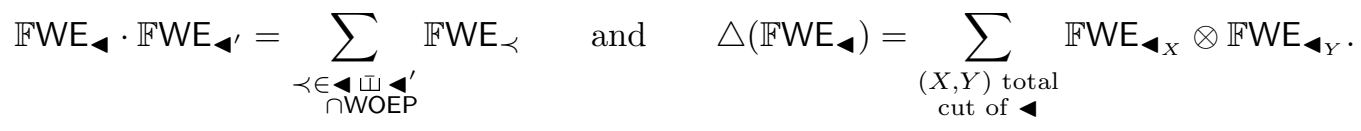

\section{Binary trees, Tamari intervals, and Schröder trees}

We now consider three families of specific integer posets corresponding to the elements (TOEP), the intervals (TOIP) and the faces (TOFP) in the Tamari order on binary trees. We construct Hopf algebras on TOEP, TOIP and TOFP as subalgebras (Section 3.4) of the integer poset Hopf algebra $(\mathcal{P}, \cdot, \triangle)$, using surjections from posets to TOEP, TOIP of TOFP whose fibers are stable by product and coproduct. We obtain Hopf algebras on TOEP (resp. TOFP) isomorphic to the Loday-Ronco Hopf algebra on binary trees [LR98] (resp. to the Chapoton Hopf algebra on Schröder trees [Cha00]), and we obtain a Hopf algebra on Tamari intervals that was not constructed earlier to the best of our knowledge.

4.1. Binary trees and the Loday-Ronco algebra. We always label the vertices of a binary tree $\mathrm{T}$ in inorder, meaning that each vertex is labeled after all vertices of its left child and before all vertices of its right child. This labeling makes $\mathrm{T}$ a binary search tree, meaning that the label of each vertex is larger than all labels in its left child and smaller than all labels in its right child. For a permutation $\sigma$, we denote by bt $(\sigma)$ the tree obtained by binary search tree insertion of $\sigma$ : it is obtained by inserting the entries of $\sigma$ from right to left such that each intermediate step remains a binary search tree. Said differently, bt $(\sigma)$ is the unique binary search tree T such that if vertex $i$ is a descendant of vertex $j$ in T, then $i$ appears before $j$ in $\sigma$. An example is illustrated in Figure 9 .

Recall that the classical Tamari lattice is the lattice on binary trees whose cover relations are the right rotations. It is also the lattice quotient of the weak order under the congruence relation given by the fibers of the binary search tree insertion. 


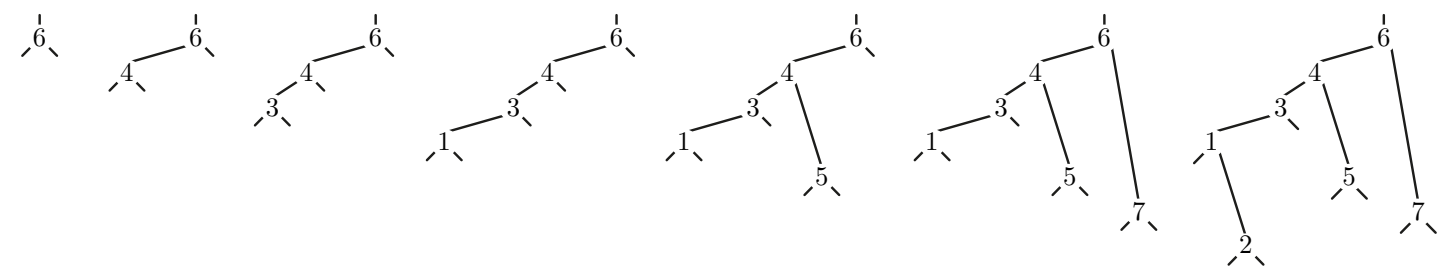

FIGURE 9. Binary search tree insertion of the permutation $\sigma=2751346$.

Finally, recall that the Loday-Ronco algebra [LR98] is the Hopf subalgebra of the MalvenutoReutenauer algebra generated by the elements

$$
\mathbb{F}_{\mathrm{T}}:=\sum_{\substack{\sigma \in \mathfrak{S} \\ \operatorname{bt}(\sigma)=\mathrm{T}}} \mathbb{F}_{\sigma}
$$

for all binary trees $\mathrm{T}$.

Example 71. For instance

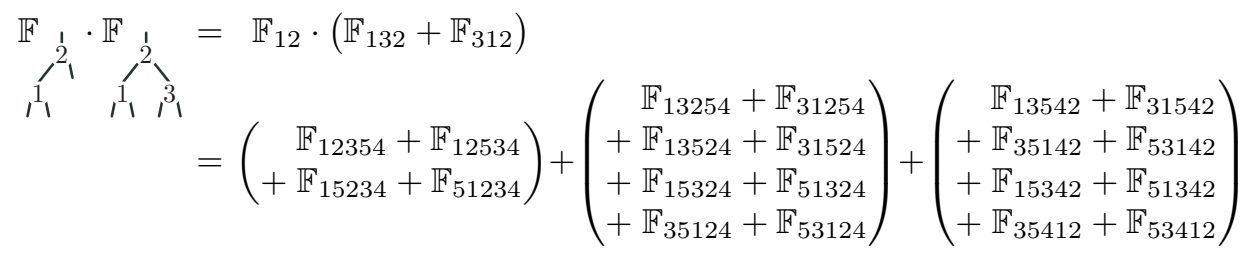

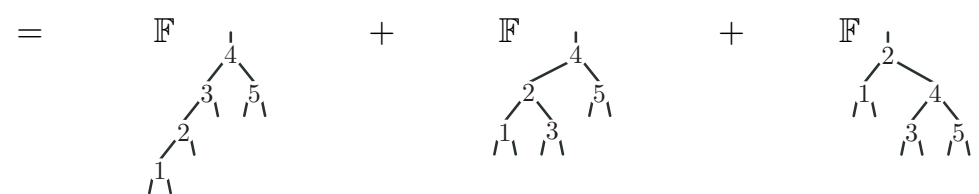

and

$$
\begin{aligned}
& \triangle\left(\mathbb{F}_{1}\right)=\triangle\left(\mathbb{F}_{132}+\mathbb{F}_{312}\right) \\
& \text { 高多 }
\end{aligned}
$$

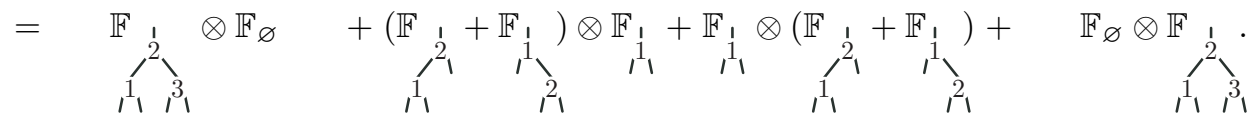

4.2. Tamari order element, interval and face posets. We now briefly recall how the elements, the intervals and the faces of the Tamari lattice can be interpreted as specific interval posets as developed in [CPP17].

4.2.1. Elements. We consider the tree $\mathrm{T}$ as the Tamari order element poset $\triangleleft_{\mathrm{T}}$ defined by $i \triangleleft_{\mathrm{T}} j$ when $i$ is a descendant of $j$ in T. In other words, the Hasse diagram of $\triangleleft_{\mathrm{T}}$ is the tree T oriented towards its root. An illustration is provided in Figure 10. Note that the increasing (resp. decreasing) subposet of $\triangleleft_{\mathrm{T}}$ is given by $i \triangleleft_{\mathrm{T}}^{\operatorname{Inc}} j$ (resp. $i \triangleleft_{\mathrm{T}}^{\mathrm{Dec}} j$ ) if and only if $i$ belongs to the left (resp. right) subtree of $j$ in T.

We define

$$
\operatorname{TOEP}_{n}:=\left\{\triangleleft_{\mathrm{T}} \mid \mathrm{T} \in \mathfrak{B}_{n}\right\} \quad \text { and } \quad \text { TOEP }:=\bigsqcup_{n \in \mathbb{N}} \operatorname{TOEP}_{n} .
$$

The following statements provide a local characterization of the posets of $\mathrm{TOEP}_{n}$ and describe the weak order induced by $\operatorname{TOEP}_{n}$.

Proposition 72 ([CPP17, Prop. 39]). A poset $\triangleleft \in \mathcal{P}_{n}$ is in $\mathrm{TOEP}_{n}$ if and only if

- $\forall a<b<c, a \triangleleft c \Rightarrow b \triangleleft c$ and $a \triangleright c \Rightarrow a \triangleright b$,

- for all $a<c$ incomparable in $\triangleleft$, there exists $a<b<c$ such that $a \triangleleft b \triangleright c$. 


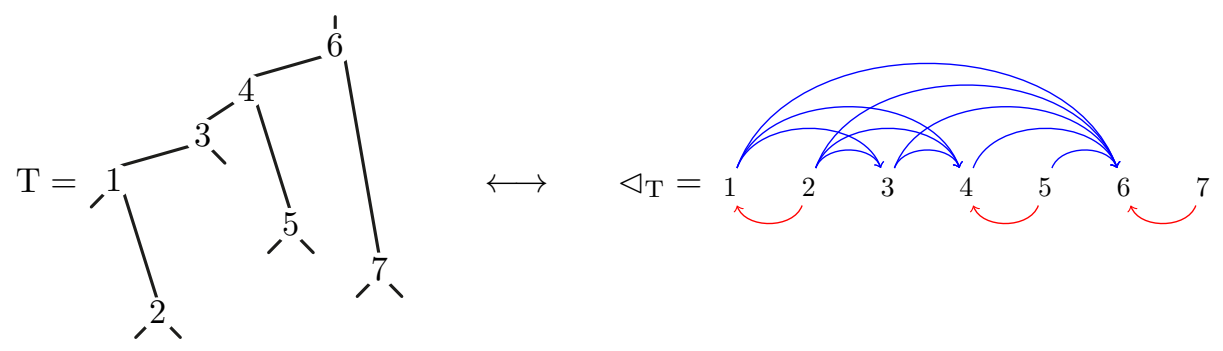

Figure 10. A Tamari Order Element Poset (TOEP).

Proposition 73 ([CPP17, Prop. 40 \& 41]). The map $\mathrm{T} \mapsto \triangleleft_{\mathrm{T}}$ is a lattice isomorphism from the Tamari lattice on binary trees of $\mathfrak{B}_{n}$ to the sublattice of the weak order on $\mathcal{P}_{n}$ induced by $\operatorname{TOEP}_{n}$.

4.2.2. Intervals. We now present a similar interpretation of the intervals of the Tamari lattice. For $\mathrm{T} \preccurlyeq \mathrm{T}^{\prime} \in \mathfrak{B}_{n}$, we consider the Tamari order interval $\left[\mathrm{T}, \mathrm{T}^{\prime}\right]:=\left\{\mathrm{S} \in \mathfrak{B}_{n} \mid \mathrm{T} \preccurlyeq \mathrm{S} \preccurlyeq \mathrm{T}^{\prime}\right\}$, and interpret it as the Tamari order interval poset $\triangleleft_{\left[\mathrm{T}, \mathrm{T}^{\prime}\right]}:=\bigcap_{\mathrm{T} \preccurlyeq \mathrm{S} \preccurlyeq \mathrm{T}^{\prime}} \triangleleft_{\mathrm{T}}=\triangleleft_{\mathrm{T}} \cap \triangleleft_{\mathrm{T}^{\prime}}=\triangleleft_{\mathrm{T}^{\prime}}^{\text {Inc }} \cap \triangleleft_{\mathrm{T}}^{\text {Dec }}$. This poset $\triangleleft_{\left[\mathrm{T}, \mathrm{T}^{\prime}\right]}$ was introduced in [CP15] with the motivation that its linear extensions are precisely the linear extensions of all binary trees in the interval $\left[T, T^{\prime}\right]$. See Figure 11.

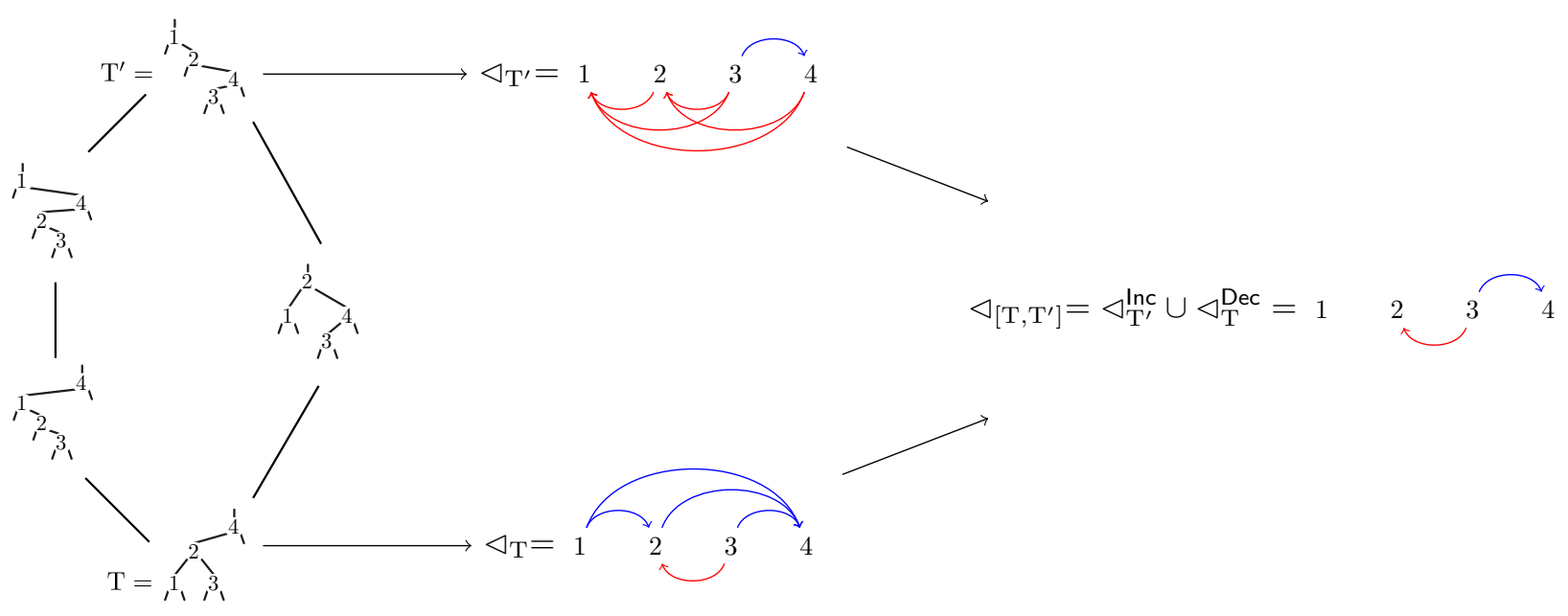

Figure 11. A Tamari Order Interval Poset (TOIP).

We define

$$
\operatorname{TOIP}_{n}:=\left\{\triangleleft_{\left[\mathrm{T}, \mathrm{T}^{\prime}\right]} \mid \mathrm{T}, \mathrm{T}^{\prime} \in \mathfrak{B}_{n}, \mathrm{~T} \preccurlyeq \mathrm{T}^{\prime}\right\} \quad \text { and } \quad \mathrm{TOIP}:=\bigsqcup_{n \in \mathbb{N}} \mathrm{TOIP}_{n} .
$$

The following statements provide a local characterization of the posets of $\mathrm{TOIP}_{n}$ and describe the weak order induced by $\mathrm{TOIP}_{n}$.

Proposition 74 ([CP15, Thm. 2.8]). A poset $\triangleleft \in \mathcal{P}(n)$ is in $\operatorname{TOIP}(n)$ if and only if $a \triangleleft c \Rightarrow b \triangleleft c$ and $a \triangleright c \Rightarrow a \triangleright b$ for all $1 \leq a<b<c \leq n$.

As in Section 3.2.2, note that this characterization clearly splits into a condition on the increasing relations and a condition on the decreasing relations of $\triangleleft$. This defines two superfamilies ITOIP ${ }_{n}$ and DTOIP of TOIP $_{n}$ with TOIP $_{n}=$ ITOIP $_{n} \cap$ DTOIP $_{n}$. 
Proposition 75 ([CPP17, Prop. $40 \& 41]$ ).

(i) If $\mathrm{S} \preccurlyeq \mathrm{S}^{\prime}$ and $\mathrm{T} \preccurlyeq \mathrm{T}^{\prime}$ in $\mathfrak{B}_{n}$, then $\triangleleft_{\left[\mathrm{S}, \mathrm{S}^{\prime}\right]} \preccurlyeq \triangleleft_{\left[\mathrm{T}, \mathrm{T}^{\prime}\right]} \Longleftrightarrow \mathrm{S} \preccurlyeq \mathrm{T}$ and $\mathrm{S}^{\prime} \preccurlyeq \mathrm{T}^{\prime}$.

(ii) The weak order on $\mathrm{TOIP}_{n}$ is a lattice whose meet $\triangleleft_{\left[\mathrm{S}, \mathrm{S}^{\prime}\right]} \wedge_{\mathrm{TOIP}} \triangleleft_{\left[\mathrm{T}, \mathrm{T}^{\prime}\right]}=\triangleleft_{\left[\mathrm{S} \wedge_{\mathfrak{B}} \mathrm{T}, \mathrm{S}^{\prime} \wedge_{\mathfrak{B}} \mathrm{T}^{\prime}\right]}$ and join $\triangleleft_{\left[\mathrm{S}, \mathrm{S}^{\prime}\right]} \vee_{\mathrm{TOIP}} \triangleleft_{\left[\mathrm{T}, \mathrm{T}^{\prime}\right]}=\triangleleft_{\left[\mathrm{S}_{\mathfrak{B}} \mathrm{T}, \mathrm{S}^{\prime} \vee_{\mathfrak{B}} \mathrm{T}^{\prime}\right]}$. Moreover, the weak order on $\mathrm{TOIP}_{n}$ is a sublattice of the weak order on $\mathcal{P}_{n}$.

4.2.3. Faces. Consider now a face of the associahedron, that is, a Schröder tree S (a rooted tree where each internal node has at least two children). We label the angles between two consecutive children in inorder, meaning that each angle is labeled after the angles in its left child and before the angles in its right child. We associate to $\mathrm{S}$ the poset $\triangleleft_{\mathrm{S}}$ where $i \triangleleft_{\mathrm{S}} j$ if and only if the angle labeled $i$ belongs to the left or to the right child of the angle labeled $j$. See Figure 12.

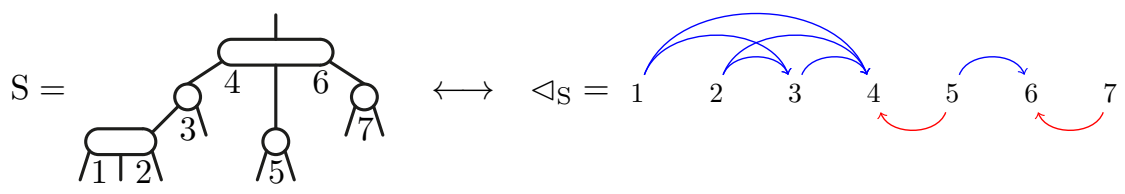

Figure 12. A Tamari Order Face Poset (TOFP).

We define

$$
\operatorname{TOFP}_{n}:=\left\{\triangleleft_{\mathrm{S}} \mid \mathrm{S} \text { Schröder tree on }[n]\right\} \quad \text { and } \quad \text { TOFP }:=\bigsqcup_{n \in \mathbb{N}} \operatorname{TOFP}_{n} .
$$

The following statements provide a local characterization of the posets of $\mathrm{TOFP}_{n}$ and describe the weak order induced by TOFP ${ }_{n}$.

Proposition 76 ([CPP17, Prop. 65]). A poset $\triangleleft$ is in $\mathrm{TOFP}_{n}$ if and only if $\triangleleft \in \mathrm{TOIP}_{n}$ and for all $a<c$ incomparable in $\triangleleft$, either there exists $a<b<c$ such that $a \not b \rtimes c$, or for all $a<b<c$ we have $a \triangleright b \triangleleft c$.

Proposition 77 ([CPP17, Sect. 2.2.3]).

(1) For any Schröder trees $\mathrm{S}, \mathrm{S}^{\prime}$, we have $\triangleleft_{\mathrm{S}} \preccurlyeq \triangleleft_{\mathrm{S}^{\prime}} \Longleftrightarrow \mathrm{S} \preccurlyeq \mathrm{S}^{\prime}$ in the facial weak order on the associahedron Asso( $n)$ studied in [PR06, DHP18]. This order is a quotient of the facial weak order on the permutahedron by the fibers of the Schröder tree insertion st.

(2) The weak order on $\mathrm{TOFP}_{n}$ is a lattice but not a sublatice of the weak order on $\mathcal{P}_{n}$, nor on $\mathrm{WOIP}_{n}$, nor on $\mathrm{TOIP}_{n}$.

To conclude, let us recall that there is a natural Schröder tree insertion map from ordered partitions to Schröder trees, similar to the binary search tree insertion from permutations to binary trees. The Schröder tree $\operatorname{st}(\pi)$ obtained from an ordered partition $\pi$ is the unique Schröder tree such that if $i$ is a descendant of $j$ (meaning that $i$ appears in a vertex that is a descendant of the vertex containing $j$ ), then $i$ appears before $j$ in $\pi$. See [Cha00, CP17, PP18] for details and Figure 13 for an illustration.
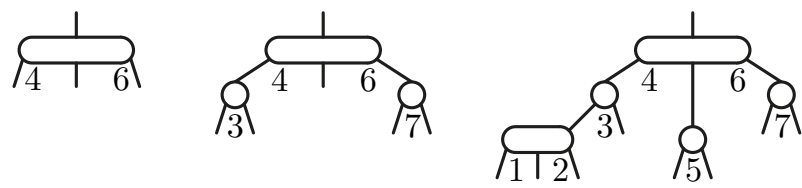

FiguRE 13. Schröder tree insertion of the ordered partition $\pi=125|37| 46$. 
4.3. Quotient algebras. Contrarily to Section 3.3, a naive construction of quotient Hopf algebras cannot directly work for TOEP, TOIP and TOFP. Although they clearly define quotient algebras, they do not define quotient cogebras since the convolution does nos satisfy a property similar to (ii) of Proposition 45: an element not in TOEP (resp. TOIP and TOFP) can appear in the convolution product of two elements of TOEP (resp. TOIP and TOFP) as in the following example:

$$
\overbrace{22}^{2} 3 \in 1 \star 1 \varkappa^{2}
$$

Indeed, see that the element on the left is neither a TOEP, TOIP nor a TOFP but it belongs to the convolution of the two integer posets on the right, which are both TOEP, TOIP and TOFP.

4.4. Subalgebras. We now construct Hopf algebras on TOEP, TOIP and TOFP as subalgebras of the Hopf algebras kWOEP, kWOIP and kWOFP respectively. For this, we need the TOIP deletion defined in [CPP17, Sect. 2.2.4] by

$$
\triangleleft^{\mathrm{TOIPd}}:=\triangleleft \backslash(\{(a, c) \mid \exists a<b<c, b \not c\} \cup\{(c, a) \mid \exists a<b<c, a \not b\}) .
$$

This operation is illustrated on Figure 14.

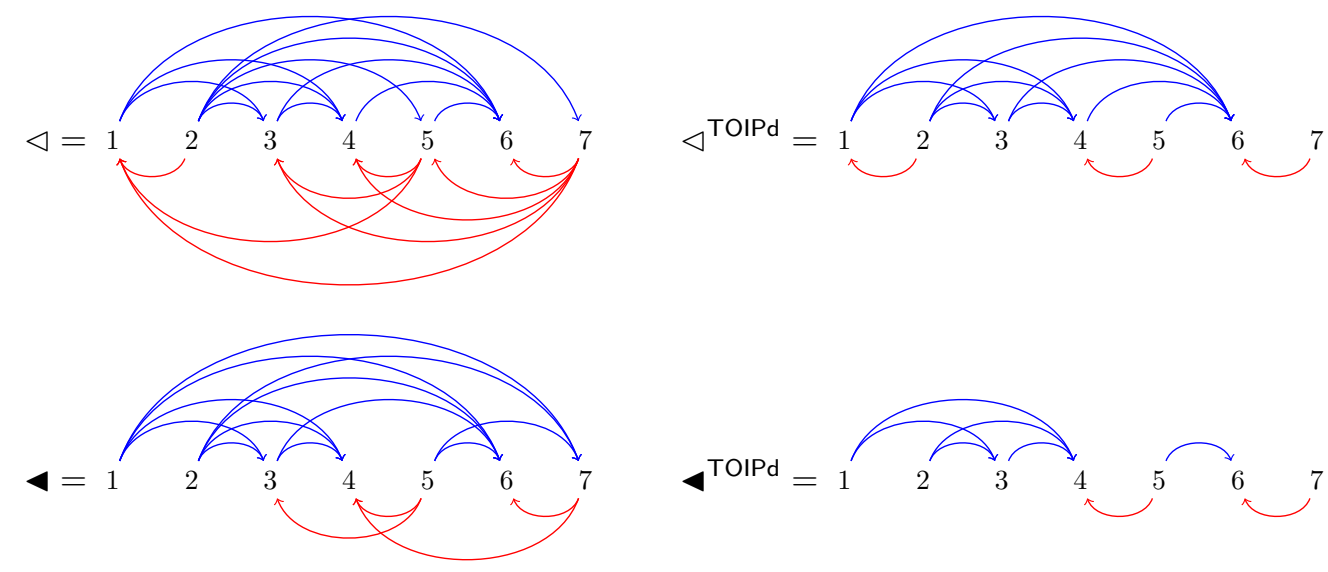

Figure 14. The TOIP deletion.

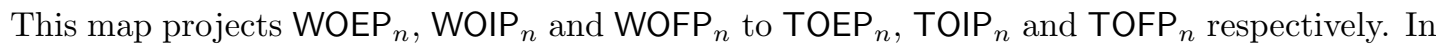
fact, it is a simple generalization of both the binary tree insertion and the Schröder tree insertion.

Proposition 78 ([CPP17, Prop. 48]). For any permutation $\sigma$, any weak order interval $\sigma \preccurlyeq \sigma^{\prime}$, and any ordered partition $\pi$, we have

$$
\left(\triangleleft_{\sigma}\right)^{\mathrm{TOIPd}}=\triangleleft_{\mathrm{bt}(\sigma)}, \quad\left(\triangleleft_{\left[\sigma, \sigma^{\prime}\right]}\right)^{\mathrm{TOIPd}}=\triangleleft_{\left[\mathrm{bt}(\sigma), \mathrm{bt}\left(\sigma^{\prime}\right)\right]} \quad \text { and } \quad\left(\triangleleft_{\pi}\right)^{\mathrm{TOIPd}}=\triangleleft_{\mathrm{st}(\pi)},
$$

where $\operatorname{bt}(\sigma)$ is the binary tree insertion of the permutation $\sigma$ and $\operatorname{st}(\pi)$ is the Schröder tree insertion of the ordered partition $\pi$.

Example 79. Compare Figures 4 and 10, Figures 5 and 11, and Figures 7 and 12.

We now use this map $\triangleleft \mapsto \triangleleft^{\mathrm{TO} P \mathrm{Pd}}$, mimicking the construction of the Loday-Ronco algebra on binary trees [LR98, HNT05] as a Hopf subalgebra of the Malvenuto-Reutenauer algebra on permutations [MR95]. For $\triangleleft \in$ TOIP, consider the element

$$
\mathbb{F T I}_{\triangleleft}:=\sum \mathbb{F W I} \mathrm{J}_{\triangleleft}
$$


where the sum runs over all $\triangleleft \in$ WOIP such that $\triangleleft^{\mathrm{TOIPd}}=\mathbf{4}$. We denote by $\mathbf{k T O I P}$ the linear subspace of $\mathbf{k} \mathcal{P}$ spanned by the elements $\mathbb{F T I}_{\mathbf{\triangleleft}}$ for $\boldsymbol{\iota} \in$ TOIP. Similarly, we define the linear subspace kTOEP (resp. kTOFP) spanned by the elements

$$
\mathbb{F T E}_{\triangleleft}:=\sum \mathbb{F W E} \mathrm{E}_{\triangleleft} \quad\left(\text { resp. } \mathbb{F T E} \triangleleft:=\sum \mathbb{F W E} \mathrm{E}_{\triangleleft}\right)
$$

for all $\triangleleft \in$ TOEP (resp. TOFP) where the sum runs over all $\triangleleft \in$ WOEP (resp. WOFP) such that $\triangleleft^{\mathrm{TOIPd}}=4$.

Remark 80. Note that the fiber of a TOEP (resp. TOFP) under the map $\triangleleft \mapsto \triangleleft^{\text {TOIPd }}$ is not in WOEP (resp. WOFP) in general. For example, $\triangleleft_{[132,312]}=\widetilde{r}_{1}^{r_{2}} 3$ is not in WOEP, but

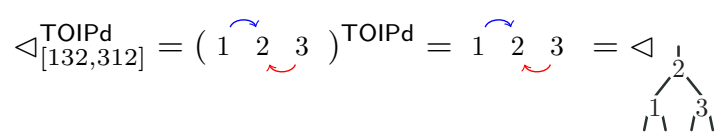

is in TOEP. The element $\mathbb{F T E}_{\triangleleft}($ resp. $\mathbb{F T F} \triangleleft)$ is defined as the sum over the fiber of $\triangleleft$ in WOEP (resp. in WOFP).

Example 81. Here is an example of computation of a product and a coproduct of elements $F T E_{4}$ computed in the algebra kWOEP. In the second line, for each element $\mathbb{F W E} \triangleleft$ of the sum in $\mathrm{kWOEP}$, we have bolded the subrelation $\triangleleft^{\text {TOIPd }}$. Observe that the result can again be expressed in the basis $\mathbb{F T E} \triangleleft$ as will be proven in Proposition 84 .

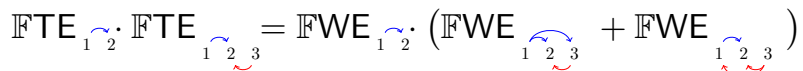

$$
\begin{aligned}
& =(\mathbb{F W E}+F W E
\end{aligned}
$$

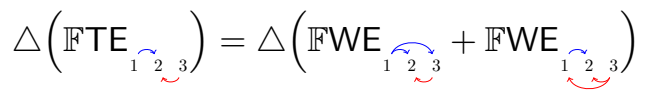

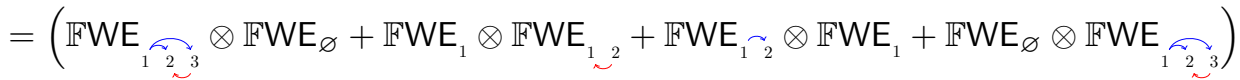

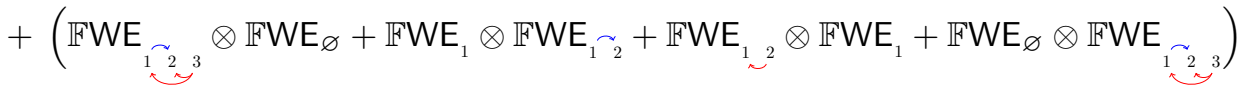

$$
\begin{aligned}
& =\mathbb{F} \mathrm{TE}_{\sim_{\sim_{2} \sim_{3}}} \otimes \mathbb{F} \mathrm{TE} \mathrm{E}_{\varnothing}+\mathbb{F} \mathrm{TE}_{1} \otimes\left(\mathbb{F T E}_{\sim_{\sim^{2}}}+\mathbb{F} \mathrm{TE}_{\sim_{\sim_{2}}}\right)
\end{aligned}
$$

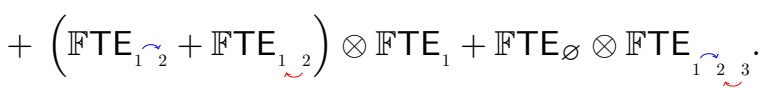

Example 82. Here is an example of computation of a product and a coproduct of elements $\mathbb{F T I}_{4}$ computed in the algebra kWOIP. In the second line, for each element $\mathbb{F W I}_{\triangleleft}$ of the sum in kWOIP, we have bolded the subrelation $\triangleleft^{\text {TOIPd }}$. Observe that the result can again be expressed in the basis $\mathbb{F T I}_{\triangleleft}$ as will be proven in Proposition 84 . 


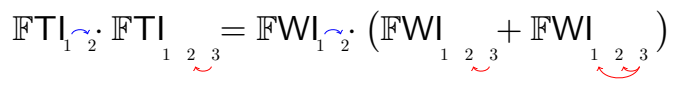

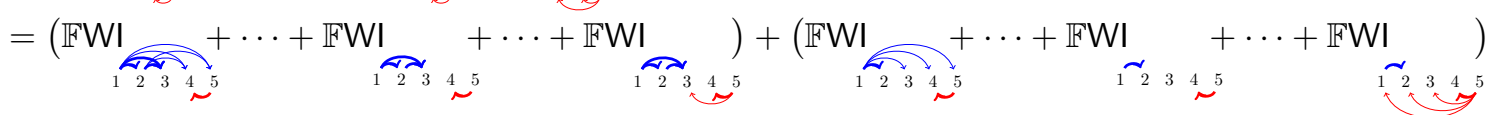

$$
\begin{aligned}
& +(\mathbb{F W I} \overbrace{\sim_{2}^{4}}+\cdots+\mathbb{F W I}+\cdots+\mathbb{F W I})+(\mathbb{F W I}+\cdots+\mathbb{F W I}+\cdots+\mathbb{F W I})
\end{aligned}
$$

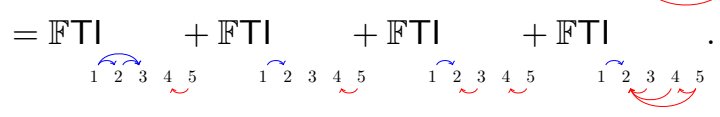

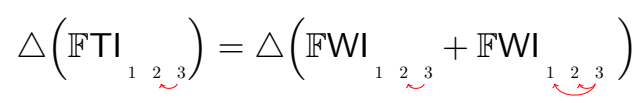

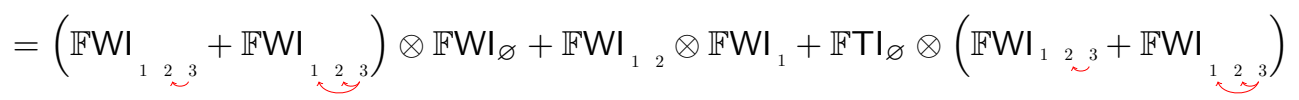

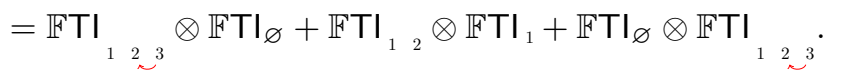

Example 83. Here is an example of computation of a product and a coproduct of elements FTF computed in the algebra kWOFP. In the second line, for each element $\mathbb{F W F} \triangleleft$ of the sum in kWOFP, we have bolded the subrelation $\triangleleft^{\mathrm{TOIPd}}$. Observe that the result can again be expressed in the basis $\mathbb{F} T F_{\triangleleft}$ as will be proven in Proposition 84 .

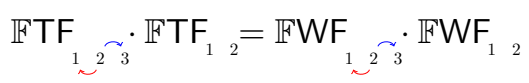

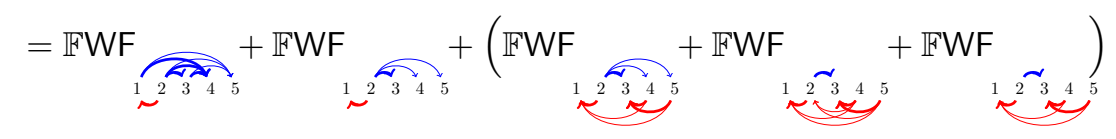

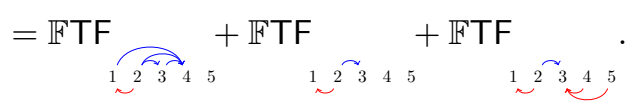

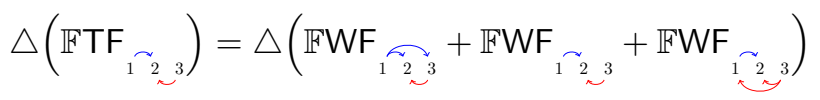

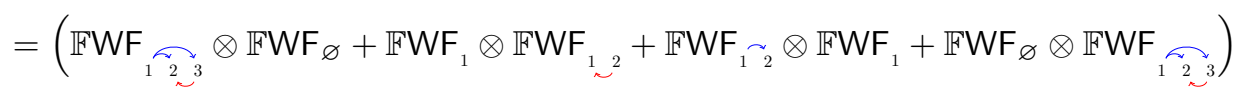

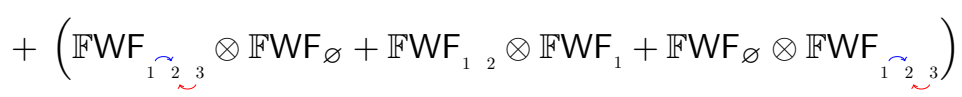

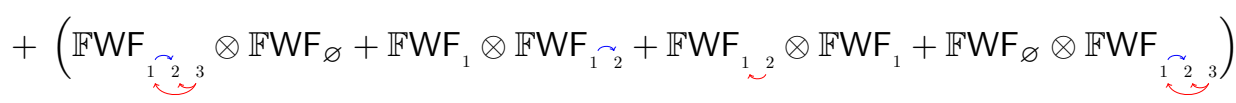

$$
\begin{aligned}
& =\mathbb{F} T F_{r_{2_{2} 3}} \otimes \mathbb{F} T F_{\varnothing}+\mathbb{F} T F_{1} \otimes \mathbb{F T F}_{1_{\sim^{2}}}+\mathbb{F T F}_{r_{2}} \otimes \mathbb{F T F}_{1}+\mathbb{F T F}_{1_{2}} \otimes \mathbb{F} \mathrm{TF}_{1} \\
& +\mathbb{F} \mathrm{TF}_{1} \otimes \mathbb{F} \mathrm{TF}_{\widetilde{1}_{2}}+\mathbb{F} \mathrm{TF}_{\sim_{\sim^{2}}} \otimes \mathbb{F} \mathrm{TF}_{1}+\mathbb{F} \mathrm{TF}_{\varnothing} \otimes \mathbb{F T F}_{\widetilde{1}_{2_{\sim_{3}}}} .
\end{aligned}
$$

Proposition 84. The subspace $\mathbf{k T O I P}$ is stable by the product $\cdot$ and the coproduct $\triangle$ and thus defines a Hopf subalgebra of $(\mathbf{k W O I P}, \cdot, \triangle)$. A similar statement holds for TOEP and TOFP.

Proof. We start with the product. Consider $\triangleleft \in \mathrm{TOIP}_{m}$ and $\boldsymbol{\iota}^{\prime} \in \mathrm{TOIP}_{n}$ and define

- $U$ as the set of WOIP's of the form $\triangleleft \sqcup \overline{\triangleleft^{\prime}} \sqcup \mathrm{I} \sqcup \mathrm{D}$ for any $\triangleleft \in \mathrm{WOIP}_{m}$ with $\triangleleft^{\mathrm{TOIPd}}=$ any $\triangleleft^{\prime} \in$ WOIP $_{n}$ with $\triangleleft^{\prime \text { TOIPd }}=\triangleleft^{\prime}$, any $\mathrm{I} \subseteq[m] \times \overline{[n]}$ and any D $\subseteq \overline{[n]} \times[m]$.

- $V$ as the set of TOIP's of the form $\sqcup \sqcup \triangleleft^{\prime} \sqcup \mathrm{I} \sqcup \mathrm{D}$ for any $\mathrm{I} \subseteq[\mathrm{m}] \times \overline{[n]}$ and $\mathrm{D} \subseteq \overline{[n]} \times[m]$. Note that $\dashv \in U$ if and only if $\dashv^{\mathrm{TOIPd}} \in V$. Since the product $\mathbb{F T I}_{\boldsymbol{\triangleleft}} \cdot \mathbb{F T I}_{\boldsymbol{\triangleleft}}$, contains exactly all the posets of $U$, we conclude that a poset appears in $\mathbb{F T I}_{\triangleleft} \cdot \mathbb{F T I}_{\triangleleft}$ if and only if all posets in its TOIP deletion fiber do. Therefore, the product $\mathbb{F T I}_{\triangleleft} \cdot \mathbb{F T I} \mathbf{\iota}^{\prime}$ belongs to the subspace $\mathbf{k T O I P}$. 
We now deal with the coproduct. Consider $\varangle \in \operatorname{TOIP}_{p}$ and a partition $[p]=X \sqcup Y$. Consider the set $U_{X, Y}$ of WOIP's of the form $\triangleleft \cup \triangleleft^{\prime} \cup(X \times Y)$ where $\triangleleft \subseteq X^{2}$ is such that $\left(\triangleleft_{X}\right)^{\text {TOIPd }}=\triangleleft_{X}$ and $\triangleleft^{\prime} \subseteq Y^{2}$ is such that $\left(\triangleleft_{Y}^{\prime}\right)^{\text {TOIPd }}=\triangleleft_{Y}$. Note that either none or all $\dashv \in U_{X, Y}$ satisfy $\dashv^{\text {TOIPd }}=4$. Since the coproduct $\triangle\left(\mathbb{F} T I_{\triangleleft}\right)$ contains exactly all tensors $\triangleleft_{X} \otimes \triangleleft_{Y}^{\prime}$ such that $\triangleleft \cup \triangleleft^{\prime} \cup(X \times Y) \in U_{X, Y}$ for all partitions $[p]=X \sqcup Y$, we conclude that a tensor $\triangleleft_{X} \otimes \triangleleft_{Y}^{\prime}$ appears in $\triangle(\mathbb{F T I}$ ४ if and only if all the tensors $\dashv_{X} \otimes \dashv_{Y}^{\prime}$ with $\dashv^{\text {TOIPd }}=\triangleleft^{\text {TOIPd }}$ and $\dashv^{\prime \text { TOIPd }}=\triangleleft^{\prime \text { TOIPd }}$ appear in $\triangle\left(\mathbb{F T I}_{\triangleleft}\right)$ as well. Therefore, the coproduct $\triangle\left(\mathbb{F T I}_{\triangleleft}\right)$ belongs to the subspace $\mathbf{k T O I P}$.

Proposition 85. For $\triangleleft \in \mathrm{TOIP}_{m}$ and $\triangleleft \in \mathrm{TOIP}_{n}$, the product $\mathbb{F P}_{\triangleleft} \cdot \mathbb{F} \mathcal{P}_{\triangleleft}$ is the sum of $\mathbb{F} \mathcal{P}_{\dashv}$, where $\dashv$ runs over the interval between $\triangleleft \backslash \triangleleft$ and $\triangleleft / \triangleleft$ in the weak order on $\mathrm{TOIP}_{m+n}$. A similar statement holds for TOEP and TOFP.

Proof. It is a direct consequence of Proposition 6 and the fact that for any two Tamari order element (resp. interval, resp. face) posets $\triangleleft, \triangleleft \in$ WOIP, the relations $\triangleleft \backslash \triangleleft$ and $\triangleleft / \triangleleft$ are both Tamari order element (resp. interval, resp. face) posets.

To the best of our knowledge, Proposition 84 provides the first Hopf structure on intervals of the Tamari lattice. Our next example illustrates the product and coproduct in this Hopf algebra on Tamari intervals, recasting Example 82 in terms of Tamari intervals.

Example 86. For instance,

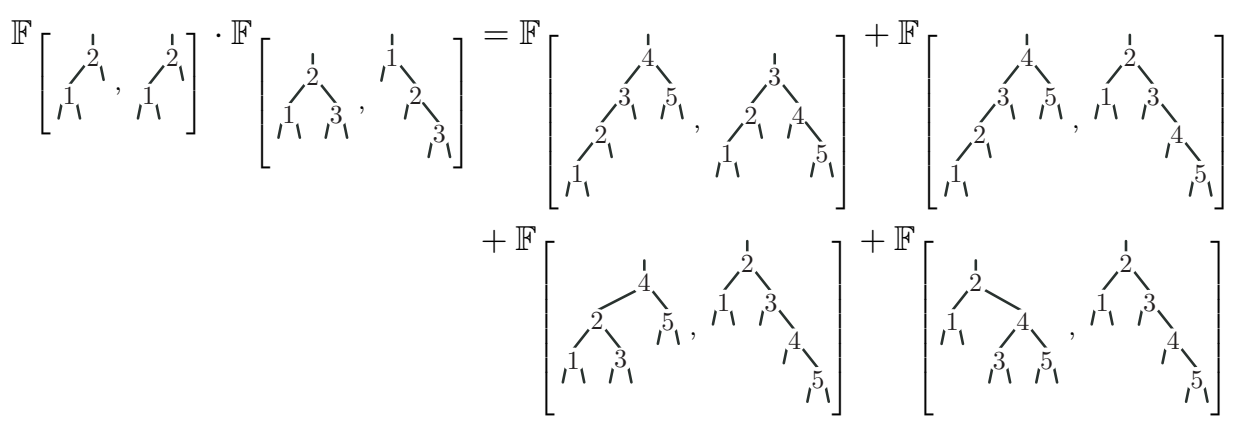

and

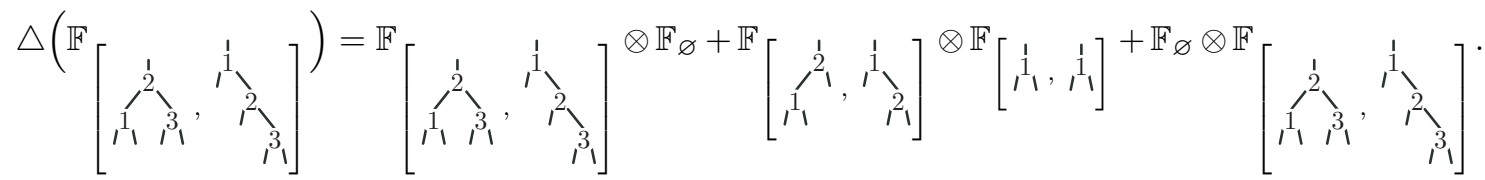

In contrast, for elements and faces, the Hopf structures of Proposition 84 already appear in the literature. We have seen already in Section 4.1 that the Loday-Ronco Hopf algebra on binary trees is the Hopf subalgebra of the Malvenuto-Reutenauer Hopf algebra on permutations generated by the sums over the fibers of the binary search tree insertion $\sigma \mapsto \operatorname{bt}(\sigma)$. Similarly, F. Chapoton defined in [Cha00] a Hopf algebra on Schröder trees obtained as a Hopf subalgebra of his Hopf algebra on ordered partitions generated by the sums over the fibers of the Schröder tree insertion $\pi \mapsto \operatorname{st}(\pi)$. We refer to [Cha00] for more details and just provide an example of product and coproduct in this Hopf algebra.

Example 87. For instance,

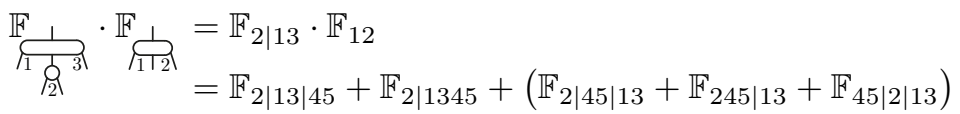

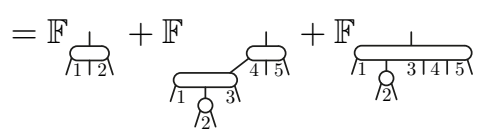


and

$$
\begin{aligned}
& \triangle\left(\mathbb{F}_{\text {d }}\right)=\triangle\left(\mathbb{F}_{1|3| 2}+\mathbb{F}_{13 \mid 2}+\mathbb{F}_{3|1| 2}+\right) \\
& =\left(\mathbb{F}_{1|3| 2} \otimes \mathbb{F}_{\varnothing}+\mathbb{F}_{1} \otimes \mathbb{F}_{2 \mid 1}+\mathbb{F}_{1 \mid 2} \otimes \mathbb{F}_{1}+\mathbb{F}_{\varnothing} \otimes \mathbb{F}_{1|3| 2}\right) \\
& +\left(\mathbb{F}_{13 \mid 2} \otimes \mathbb{F}_{\varnothing}+\mathbb{F}_{12} \otimes \mathbb{F}_{1}+\mathbb{F}_{\varnothing} \otimes \mathbb{F}_{13 \mid 2}\right) \\
& +\left(\mathbb{F}_{3|1| 2} \otimes \mathbb{F}_{\varnothing}+\mathbb{F}_{1} \otimes \mathbb{F}_{1 \mid 2}+\mathbb{F}_{2 \mid 1} \otimes \mathbb{F}_{1}+\mathbb{F}_{\varnothing} \otimes \mathbb{F}_{3|1| 2}\right)
\end{aligned}
$$

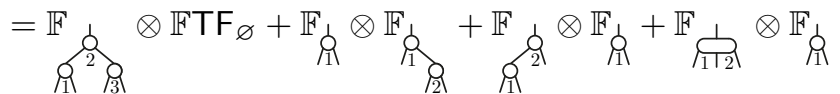

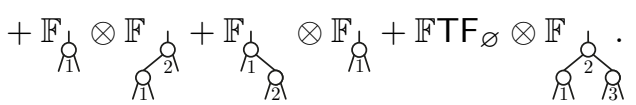

Proposition 88. - The map $\mathrm{T} \mapsto \triangleleft_{\mathrm{T}}$ is a Hopf algebra isomorphism from the Loday-Ronco algebra on binary trees [LR98, HNT05] to $(\mathbf{k T O E P}, \cdot, \triangle)$.

- The map $\mathrm{S} \mapsto \triangleleft_{\mathrm{S}}$ is a Hopf algebra isomorphism from the Chapoton algebra on Schröder trees $[$ Cha00] to $(\mathbf{k T O F P}, \cdot, \triangle)$.

Proof. This immediately follow from Propositions 48, 59 and 78.

Example 89. Compare Examples 71 and 81, and Examples 87 and 83.

Remark 90. To conclude, let us mention that similar ideas can be used to uniformly construct Hopf algebra structures on permutrees, permutree intervals, and Schröder permutrees as defined in [PP18]. Following [CP17, PP18], one first defines some decorated versions of the Hopf algebras kWOEP, kWOIP and kWOFP, where each poset on $[n]$ appears $4^{n}$ times with all possible different orientations. One then constructs Hopf algebras on kPEP, kPIP and kPFP using the fibers of the surjective map $(\triangleleft, \mathbb{O}) \mapsto \triangleleft^{\mathrm{PIP} \mathbb{O} O d}$ defined in [CPP17]. See [PP18] for details.

\section{ACKNOWLEDGMENTS}

The computation and tests needed along the research were done using the open-source mathematical software Sage [Sd16] and its combinatorics features developed by the Sage-combinat community [SCc16].

\section{REFERENCES}

[BW91] Anders Björner and Michelle L. Wachs. Permutation statistics and linear extensions of posets. J. Combin. Theory Ser. A, 58(1):85-114, 1991.

[Cha00] Frédéric Chapoton. Algèbres de Hopf des permutahèdres, associahèdres et hypercubes. Adv. Math., 150(2):264-275, 2000.

[CP15] Grégory Châtel and Viviane Pons. Counting smaller elements in the Tamari and $m$-Tamari lattices. $J$. Combin. Theory Ser. A, 134:58-97, 2015.

[CP17] Grégory Chatel and Vincent Pilaud. Cambrian Hopf Algebras. Adv. Math., 311:598-633, 2017.

[CPP17] Grégory Châtel, Vincent Pilaud, and Viviane Pons. The weak order on integer posets. Preprint, arXiv: $1701.07995,2017$.

[DHP18] Aram Dermenjian, Christophe Hohlweg, and Vincent Pilaud. The facial weak order and its lattice quotients. Trans. Amer. Math. Soc., 370(2):1469-1507, 2018.

[HNT05] Florent Hivert, Jean-Christophe Novelli, and Jean-Yves Thibon. The algebra of binary search trees. Theoret. Comput. Sci., 339(1):129-165, 2005.

$\left[\mathrm{KLN}^{+} 01\right]$ Daniel Krob, Matthieu Latapy, Jean-Christophe Novelli, Ha-Duong Phan, and Sylviane Schwer. PseudoPermutations I: First Combinatorial and Lattice Properties. 13th International Conference on Formal Power Series and Algebraic Combinatorics (FPSAC 2001), 2001.

[LR98] Jean-Louis Loday and María O. Ronco. Hopf algebra of the planar binary trees. Adv. Math., 139(2):293309, 1998.

[LR06] J.-L. Loday and M. Ronco. On the structure of cofree Hopf algebras. Journal für die reine und angewandte Mathematik, 592:123-155, 2006.

[MR95] Claudia Malvenuto and Christophe Reutenauer. Duality between quasi-symmetric functions and the Solomon descent algebra. J. Algebra, 177(3):967-982, 1995.

[PP18] Vincent Pilaud and Viviane Pons. Permutrees. Algebraic Combinatorics, 1(2):173-224, 2018.

[PR06] Patricia Palacios and María O. Ronco. Weak Bruhat order on the set of faces of the permutohedron and the associahedron. J. Algebra, 299(2):648-678, 2006. 
[SCc16] The Sage-Combinat community. Sage-Combinat: enhancing Sage as a toolbox for computer exploration in algebraic combinatorics, 2016. http://wiki.sagemath.org/combinat.

[Sd16] The Sage developers. Sage Mathematics Software, 2016. http://www.sagemath.org.

(VPi) CNRS \& LiX, École Polytechnique, Palaiseau

E-mail address: vincent.pilaud@lix.polytechnique.fr

$U R L:$ http://www.lix.polytechnique.fr/ ${ }^{\sim}$ pilaud/

(VPo) LRI, Univ. Paris-Sud - CNRS - Centrale Supelec - Univ. Paris-Saclay

E-mail address: viviane.pons@lri.fr

$U R L:$ https://www.lri.fr/ pons/ 